\title{
Quantitative Proteomics Reveal the Protective Effects of the Combined Chinese Herbal Extracts Against Alzheimer's Disease via Modulating the Expression of Synapse-associated Proteins in Mouse Model
}

\section{Qianqian Huang}

Beijing University of Chinese Medicine Affiliated Third Hospital https://orcid.org/0000-0002-0052-4395

Haibin Zhao

Beijing University of Chinese Medicine Affiliated Dongfang Hospital

\section{Chen Zhang}

School of Life Sciences,Beijing University of Chinese Medicine

\section{Ying Hao}

School of Life Sciences,Beijing University of Chinese Medicine

\section{Qihong Ma}

School of Life Sciences, Beijing University of Chinese Medicine

\section{Shi Dong}

School of Life Sciences,Beijing University of Chinese Medicine

\section{Zimin Liu}

Chenland Nutritionals, Irvine,CA

\section{Shanglong Wang}

Chenland Nutritionals, Irvine,CA

Yuanyuan Shi ( $\nabla$ yshi@bucm.edu.cn )

Beijing University of Chinese Medicine https://orcid.org/0000-0003-2997-4573

\section{Research}

Keywords: Alzheimer's Disease (AD), CuraUltra, quantitative proteomics, scopolamine-treated model mice, glutamatergic synapse

Posted Date: October 12th, 2021

DOI: https://doi.org/10.21203/rs.3.rs-956329/v1 
License: (c) (i) This work is licensed under a Creative Commons Attribution 4.0 International License. Read Full License 


\section{Abstract}

\section{Background}

Alzheimer's Disease (AD) as an age-related, irreversible neurodegenerative disease, characterized by cognitive dysfunction, has become progressively serious as a result of a global increase in life expectancy. As more mechanism of AD were discovered, therapeutic strategies using traditional Chinese medicine are under investigation for $A D$ treatment, with efforts to improve efficacy and clarify the mechanism. Gastrodia, elata Blume, Polygala tenuifolia Willd., Cistanche deserticola Ma, Rehmannia lutinosa (Gaertn.)DC.,Acorus gramineus Aiton, and Curcuma longa L. are well-known chinese herbs with neuroprotective effects and widely used as a combination in traditional Chinese decoction for $A D$ treatment. The purpose of this study was to investigate the synergistic protective efficacy of the combination (composed of extracts from these six Chinese medicines, CuraUltra), and the protein targets on scopolamine-induced cognitive impairment, using the proteomics analysis.

\section{Methods}

Scopolamine-induced cognitive impairment mouse model was established. Behavioral tests, Nissl Staining, cerebral cholinergic system alterations and neuronal apoptosis characteristics were examined to evaluate the ameliorating effects of CuraUltra on cognitive impairment induced by scopolamine. To identify the potential molecular mechanism responsible for the effect on CuraUltra treatment, label-free quantitative proteomics by tandem mass spectrometry (LC-MS/MS) were performed. Critical altered proteins were validated by qPCR and Western blotting. Molecular docking was finally performed to evaluate the binding potential of chemical components with the target proteins.

\section{Results}

Administration of CuraUltra significantly recovered scopolamine-induced cognitive impairment, as evidenced by the improved learning and memory ability, reduced pathological damage of hippocampus, increased content of Ach, decreased activity of AchE, and ameliorated expression levels of the neuronal apoptosis-related protein in the hippocampus of mice. Using quantitative proteomics technology, we observed 252 differentially expressed proteins. Compared with the model group, after CuraUltra treatments, a remarkedly alteration in PPP3CC, PKA, P38MAPK, RASA4, DNAJB1, SNAPIN was also observed. Notably, several significant proteins are in the glutamatergic synapse signaling pathway. Moreover, we confirmed that the PPP3CC appears to decreased as the AD pathological development, and may be positively correlated with the phosphorylation level of $P K A$, thereby inhibiting the activation of P38MAPK phosphorylation.

\section{Conclusions}

Administration of CuraUltra was effective on alleviating scopolamine-induced cognitive impairment, which might be through modulation of glutamatergic synapse. Consequently, our quantitative proteome 
data obtained from scopolamine-treated model mice successfully characterized AD-related biological alterations and proposed novel protein biomarkers for AD.

\section{Introduction}

Alzheimer's disease (AD) is a progressive, irreversible neurodegenerative disease characterized by learning decline and memory dysfunction with neuropathologically negative consequences. Epidemiological investigations suggest that the number of people with dementia in the world may reach 131.5 million in 2050. However, there is currently no disease-modifying therapies available for AD [1]. The cost-intensive development of drugs that target a single protein may not achieve expected therapeutic results. Traditional Chinese medicine associated with multiple targets and various pharmacological actions have obvious advantages in treating AD.

The combination of Gastrodia, elata Blume, Polygala tenuifolia Willd., Cistanche deserticola Ma, Rehmannia lutinosa (Gaertn.)DC.,Acorus gramineus Aiton is widely used in Chinese medicinal prescription for $A D$ treatment such as Tianma Xingnao capsule[2, 3], and several active components extracted from these six Chinese medicines have been proved to exhibit anti-AD properties. Curcuma, the main constituent of Curcuma longa $L$., receives much attention for its favorable neuroprotective effects for significantly preventing or treating AD [4].Gastrodin, is well known as the main functional component of the orchid plant Gastrodia elata Blume, with wide range of biological activities. Meng et al. reported that Gastrodin, has the potential to improve hippocampal neurogenesis through counteracting proinflammatory cytokines released by oligomer-induced $A \beta 42$ in a dose-dependent manner[5]. Additionally, Gastrodin was able to attenuate the altered expression levels of synaptic proteins [6]. The main active ingredients of Polygala tenuifolia Willd. include oligosaccharide esters (3,6'-disinapoyl sucrose, Sibiricose A5, Sibiricose A6), Onjisaponin, and Xanthones [7], which have been documented to have mitigative effects on neurotoxicity. For example, previous study have suggest that OnjisaponinB could contribute to the restoration of cognitive ability by improving the antioxidant and anti-inflammatory capacity in D-gal induced aging rats [8].In addition, Ethnocide, a biologically active component extracted from Cistanche deserticola $\mathrm{Ma}$, which can improved learning and memory impairment by upregulating hippocampal insulin, glucose transport, and energy metabolism [9]. Also, it was previously reported that Acteoside found in Cistanche deserticola $\mathrm{Ma}$ is able to ameliorated the deficiency of learning ability in mice induced by scopolamine [10]. Taken together, these active components extracted from these Chinese herbs may exert synergistic action against $A D$ through multiple targets and pathways. Moreover, the combination of extracts derived from these six Chinese medicines have been developed as an adjunct to dietary supplements for treating AD (CuraUltra, Batch No. CM18220191028-2, Product code: T-4010-1). It is also applied for a patent of novel formula to improve cognitive impairment (patent number:

201911113149.X). Even though there are lots of evidence of therapeutic application of CuraUltra in AD treatment, but the efficacy of the CuraUltra in AD experimental model and the further mechanism still not clearly clarified. And, the exploration of potential pharmacological mechanisms is still a challenge in the research of TCMs containing multiple herbal constituents. The powerful proteomics technology is now pushing forward the frontiers of Chinese medicine compound mechanism research. Mass spectrometry 
(MS)-based quantitative proteomics was proven to be a powerful technique to elucidate the novel pathophysiological processes and potential therapeutic targets, enabling the in-depth characterization of protein expression changes. In this study, we evaluated the potential protective effect, and explored the underlying mechanism of CuraUltra in scopolamine-induced model mice through proteomics analysis.

\section{Materials And Methods}

\subsection{Drugs and reagents}

These herbal extracts of CuraUltra were purchased from Shaanxi Jiahe Biotechnology Co., Ltd. (Shanxi China). Scopolamine hydrobromide was purchased from Beijing Bailingwei Technology Co., Ltd., (Beijing China). Batch No. LI10Q53. Huperzine A (70 mg/tablet) was purchased from Henan Tailong Pharmaceutical Co., Ltd., (Henan China). Batch No.190202. Acetylcholine assay kit and acetylcholinesterase activity assay kit was purchased from Nanjing Jian Cheng Bioengineering Institute (Jiangsu, Beijing).

\subsection{Preparation of CuraUltra}

CuraUltra formula were prepared by Gastrodia, elata Blume extract standardized to 1\% Gastrodin (Batch No. C7M-A-803456), Polygala tenuifolia Willd extract standardized to 1\% 3, 6'-Disinapoly sucrose (Batch No.CY2-A-808331), Acorus gramineus Aiton extract (10:1) (Batch No.CSCP-A-710215), Cistanche deserticola Ma extract standardized to $20 \%$ phenylethanol glycosides (Batch No.190501), Rehmannia lutinosa (Gaertn.)DC.extract (5:1) (Batch No.CSDH-A-900106), Curcuma longa L.extract standardized to $95 \%$ total curcuma (Batch No.CJH-A-908220) at the ratio of 1.25:0.67:0.18:0.3:0.125:0.1 (w/w). The ratio is based on the calculation of pharmacopoeia recommended dosage and extracts rate. the ratio of Curcuma longa $L$. extract used the low daily dose of curcumin recommended by the Chinese Pharmacopoeia (the recommended dosage is $200 \mathrm{mg}$ ). The phytochemical standardization and HPLC characteristic fingerprint for each herbal extract was completed by Shaanxi Jiahe Biotechnology Co., Ltd., (Shanxi China) (see supplementary materials).Briefly, the crude drugs of Gastrodia, elata Blume, Polygala tenuifolia Will, Cistanche deserticola Ma were pulverized to powder and sieved through a 20-mesh sieve. Gastrodia, elata Blume was extracted three times with $70 \%$ ethanol for $2 \mathrm{~h}$ each time. Polygala tenuifolia Willd was extracted three times with $80 \%$ ethanol for $2 \mathrm{~h}$ each time.Cistanche deserticola Ma was extracted three times with $50 \%$ ethanol for $2 \mathrm{~h}, 1.5 \mathrm{~h}, 1.5 \mathrm{~h}$, with a liquid-to-material ratio of $6,5,4$, respectively. Curcuma longa $L$. was extracted two times with $95 \%$ ethanol for $2 \mathrm{~h}$ each time. Acorus gramineus Aiton was soaked with 8-fold of water for $3 \mathrm{~h}$, and then extracted three times, each time for about $2 \mathrm{~h}$. Rehmannia lutinosa (Gaertn.)DC. was soaked with 8 -fold of water for $1 \mathrm{~h}$ and extracted three times for $2 h, 2 h, 1 h$, respectively. These extracts were then filtered, condensed under vacuum, freeze-dried into powder.

\subsection{LC-MS analysis of CuraUltra}


Dionex Ultimate 3000 UHPLC system equipped with a binary pump, an autosampler, a solvent degasser, and a thermostatic column compartment (Thermo Fisher, Waltham, MA, USA) were used for analysis component of CuraUltra. All chromatographic separations were performed on a Waters Acquity UPLC BDS C18 column $\left(150 \mathrm{~mm} \times 2.4 \mathrm{~mm}, 2.74\right.$ um; Thermo Fisher). The column temperature was $45^{\circ} \mathrm{C}$, the flow rate was $0.3 \mathrm{~mL} / \mathrm{min}$. The mobile phase consisted of acetonitrile $(A)$ and $0.1 \%$ formic acid aqueous solution at a flow rate of $0.3 \mathrm{~mL} / \mathrm{min}$, with gradient elution as follows: $0 \rrbracket 45 \mathrm{~min}, 95 \% \mathrm{~B} ; 45.0 \sim 45.1 \mathrm{~min}$, $25 \% \mathrm{~B} \otimes 45.1 \sim 50 \mathrm{~min}, 95 \% \mathrm{~B}$. And the column temperature was set to $45^{\circ} \mathrm{C}$. Mass Spectrometer (MS) detection was performed on a Q Exactive ${ }^{\mathrm{TM}}$ Plus mass spectrometer (Thermo Fisher Scientific, USA) equipped with an electrospray ionization source (ESI) using a positive ion spray voltage of $3.5 \mathrm{k} \mathrm{eV}$ and a negative ion spray voltage of $3.0 \mathrm{k} \mathrm{eV}$. The mass spectrometry conditions were as follows: the sheath gas and auxiliary gas was both high-purity nitrogen (purity $>99.99 \%$ ); the flow rates were 40 arbitrary units and 20 arbitrary units respectively; the capillary temperature was $320^{\circ} \mathrm{C}$; the sheath gas flow rate was $35.0 \mu \mathrm{L} / \mathrm{min}$, the aux gas flow rate was $10 \mu \mathrm{L} / \mathrm{min}$, the sweep gas flow rate was $10 \mu \mathrm{L} / \mathrm{min}$; The mass scanning range was $\mathrm{m} / \mathrm{z} 120 \otimes 1800$.

\subsection{Animals and treatment}

68 SPF male C57BL/6J mice (weight $20 \pm 2 \mathrm{~g}$ ) were used in our study. All animals were produced by Jinan Pengyue Experimental Animals Education Co., Ltd (Beijing, China). License number: SCXK (Lu) 20180003.6. The feeding procedures of animals and experimental operations have been approved by the Animal Care and Use Committee of Beijing University of Chinese Medicine (BUCM-4-2019102105-4119). Mice were housed in cages and kept under standard breeding conditions (12:12 hours light/dark cycle, controlled room temperature $\left(23 \pm 2^{\circ} \mathrm{C}\right)$, sanitary conditions, standard diets, and Stress-free environment. After adaption for 7 days, mice were randomly divided into 4 equal-sized groups: control group (Control), model group (Model), positive drug group (HupA), treatment drug group (CuraUltra), every seventeen mice were divided into one group. Mice were preventively treated in the first 30 days. For each day, Mice were administered once with CuraUltra $(416.67 \mathrm{mg} / \mathrm{kg} / \mathrm{d})$ and HuperzineA $(0.2 \mathrm{mg} / \mathrm{kg} / \mathrm{d})$ intraperitoneally (i.g.). The administration dosage of CuraUltra was calculated using dosage conversion relationship between mice and human. The previous safety evaluation (safe dose determination) showed that the maximum tolerated dose (MTD) was $26 \mathrm{~g} / \mathrm{kg}$, and no obvious abnormalities were observed. Behavioral tests were then performed to examine mouse cognitive deficits at 31, 36, 40 days, respectively, including Novel Object Recognition (NOR), Step-Down Passive Avoidance (SDA) test, Morris water maze (MWM). Therapeutic drugs were administered one hour before the behavioral test, scopolamine $(2 \mathrm{mg} / \mathrm{kg} / \mathrm{d})$ administration and normal saline were intraperitoneally injected 15-20 minutes before. The experimental period was 47 days. After the last behavioral test, mice were euthanized by cervical dislocation, the hippocampus and frontal cortex were collected, weighed, and then stored in a -80 ॰C cryo-freezer for label-free shotgun proteomics analysis and bioinformatics to provide insights on the protein responses induced by CuraUltra in the brain of $A D$ mice.

\subsection{Behavioral tests}

\subsubsection{Novel object cognition (NOR) test}


The Novel Object Recognition (NOR) test is a behavioral method that uses the nature of mice to explore strange objects to test the short-term memory ability of mice. When contacting a new object for the first time, mice will explore more frequently instead of spending time on an object they have seen before (familiar object). On the first day, place two identical objects in a room and allow the mouse to explore for 5 minutes. 24 hours later, one of the objects was replaced by a novel object in the chamber. Exploration is defined as mice touching or sniffing the object within a distance $\leq 2 \mathrm{~cm}$ from the object [11]. Recording the Total Exploration Time of Novel Object (Tn)and The Total Exploration Time of Familiar Object (TF) within 5 minutes. Calculate the Recognition Index: RI= TN/ (TN+TF)[12]. After each mouse experiment is over, clean the open field box with $75 \%$ ethanol.

\subsubsection{The step-down passive avoidance (SDA) test}

The step-down passive avoidance (SDA) test consists of a rectangular plexiglass inner box with parallel steel rods on the grid floor and a wooden platform. During the training phase, the animal was placed on the grid floor, and then the power was turn on, the electric shock lasted 5 minutes. After $24 \mathrm{~h}$, the animal was placed on the wooden platform while giving an electrical shock. The step-down latency (recorded as the time for the animals to jump-down from the platform) and the number of errors (the animals touching the grid floor with paw within 300s) were used to evaluate learning and memory performance of mice. If the mouse does not jump off the platform within 300s, the number of errors is recorded as 0 and the incubation period is recorded as 300 s.

\subsubsection{Morris water maze (MWM) test}

The Morris water maze test was performed to calculate the ability of spatial learning and memory in AD mice. The test, which lasts for 5 consecutive days, was divided into two parts, the first part was the positioning navigation experiment in the first 4 days. On the fifth day, the platform was removed for space exploration experiment[13]. During the training session, each mouse was placed into the water, facing to the wall of the pool, in one of the four quadrants. Simultaneously start with computer recording equipment. The swim time was set to $60 \mathrm{~s}$. The time spent in searing platform is called escape latency, and swimming trajectories and swimming distance of the mice were recorded within the 60s period. If the mouse failed to find the platform, alternatively, put the mouse on the platform and stand for another 15s. The platform was removed on the last day [14].

\subsection{Nissl staining}

After 30 days of treatment, the mice were anesthetized and perfused through the heart with saline and $4 \%$ formaldehyde. Subsequently, the brain tissue was removed and fixed in $4 \%$ paraformaldehyde for $48 \mathrm{~h}$. And then the brain tissue was embedded in paraffin and cut at 4- $\mu \mathrm{m}$ thickness. The images were observed by Olympus BX51 light microscopy (Olympus, Japan).

\subsection{Protein extraction and digestion}

In this experiment, there were four groups of mice ( $n=17$ per group) to evaluate the proteome treated by CuraUltra. In each group, 9 hippocampus samples isolated from different animals were pooled into three 
samples for proteomic analyses, followed by protein extraction and quantification. The proteomics analysis protocol has been reported previously $[15,16]$. The proteins were extracted with the mammalian tissue total protein extraction kit (AP0601-50, Bang Fei Bioscience Co, Ltd, Beijing, China), and the protein concentration was determined using the protein quantification kit according to the manufacturer's instructions. Approximately $25 \mathrm{ug}$ of brain tissues was homogenized in $250 \mathrm{ul}$ of extraction buffer for 2 min using a homogenizer. Then, the lysates were centrifuged at $20,000 \times \mathrm{g}$ for $10 \mathrm{~min}$ at $4^{\circ} \mathrm{C}$. Next, the supernatant was recovered. The protein concentration was measured by BCA (bicinchoninic acid) kit (Thermo Fisher Scientific, America) according to the manufacturer's protocol. After protein quantification, Protein solution samples were prepared [15]. Then, the samples were digested with trypsin following the Filter Aided Sample Preparation (FASP) protocol. All the peptide samples were collected for mass spectrometry analysis.

\subsection{Label free quantitative proteomic analyses}

Each sample was separated using a nanoliter flow rate Easy nLC1000 system. Digested peptide mixtures were pressure-loaded onto a C18 trapping column (Thermo Fisher Scientific, Acclaim PepMapRSLC 50um $X 15 \mathrm{~cm}$, nano viper, $P / N 164943$ ), and the column was washed with buffer $A$ (water, $0.1 \%$ formic acid) and buffer $B(80 \%$ acetonitrile and $0.1 \%$ formic acid) at a flow rate of $600 \mathrm{~nL} / \mathrm{min}$. After chromatographic separation, LC-MS/MS analysis was conducted using Q Exactive HF-X mass spectrometer (Thermo Scientific) that was coupled with Easy nLC (Thermo Fisher Scientific) for $88 \mathrm{~min}$. The mass spectrometer was operated in positive ion mode with MS1 survey scan (m/z: 350-1550). The resolution of the primary mass spectrum was 120,000 , Automatic gain control (AGC) target was set to $3 \mathrm{e} 6$, and the maximum time of the primary ion was $20 \mathrm{~ms}$. Normalized collision energy was $30 \mathrm{eV}$.

\subsection{Mass spectrometry data analysis and bioinformatics analysis}

Peptide targeted identification was conducted with the SEQUST search engine, using sequences of human proteins downloaded from Uniprot [15]. All peptide raw files were analyzed by the Proteome Discover (2.2.0.388) and compared against the Uniprot Mus protein database (uniprotMus+musculus_20190102.fasta). Results were filtered with Peptide false discovery rate (FDR) $\leq 0.01$. The following parameters were used in this analysis: Enzyme, Trypsin; Max Missed Cleavages, 2; Peptide Mass Tolerance, \pm 15 ppm; Fixed modifications, Carbamidomethyl (C); Variable modifications, Oxidation (M); Acetyl (Protein N-term); Fragment Mass Tolerance, $20 \mathrm{mmu}$; peptide confidence, high. The upregulated or downregulated proteins in both replicates with relative quantification $p$-values $<0.05$ and 1.2 fold-changes (FC) were selected as being differentially expressed in the data. We performed functional analysis of altered protein with Gene Ontology-term annotations and KEGG pathway analysis using DAVID and visualized the results with CLUGO plug-in cytoscape 3.6.0 software. Based on the GO and KEGG analyses, The protein-protein interaction (PPI) networks were performed using String database and interpreted with cytoscape. 


\subsection{Measurement of acetylcholinesterase (AchE) activity and acetylcholine (Ach) Content from the mice brain}

The level of Ach and AchE in the hippocampus ( $n=3$ mice per group) homogenates were detected by using commercially available kit. The tissues were then processed according to the manufacturer's instructions of the Ach assay kit (CAT: A105-1-1) and AchE activity assay kit (CAT: A105-2-1).

- 2.11 RT-qPCR analysis

Total RNA was extracted from hippocampus tissue using RNeasy® Lipid Tissue Mini Kit (QIAGEN, Valencia, CA, USA). The quality and quantity of RNA were measured using spectrophotometer (Thermo Nano Drop ${ }^{\text {TM }} 2000$ c, USA). We used the following primers: Forward primer-PPP3CC TGCACACAGGATCCGAAGTT, Reverse primer-PPP3CC CTTTCGGGGTGGCATTCTCTC. Forward primerSNAPIN GCCGGATCAATGAGGATCAG, Reverse primer-SNAPIN TTGACCAAGACAACTCGTCG. Forward primer-GAPDH AGGTTGTCTCCTGCGACTTCA, Reverse primer-GAPDH TGGTCCAGGGTTTCTTACTCC. RNA $(2 \mu \mathrm{g})$ was converted into complementary DNA using Reverse Transcription Master Mix (QIAGEN, Valencia, CA, USA). RT-qPCR was determined using SYBR Green Master mix on a CFX-96 system (QIAGEN, Valencia, CA, USA). We used the $2^{\wedge}{ }^{\wedge}$-delta Ct method for the quantification of samples. GAPDH gene was chosen as the endogenous control to normalize variance between samples.

- 2.12 Western blotting

Total protein was extracted from frozen Brain tissue using RIPA Lysis Buffer (Applygen Technology Co., Ltd., Beijing, China) containing protease inhibitor and a phosphatase inhibitor. Protein concentration from tissue homogenates was measured with a BCA protein assay kit (Applygen, Beijing, China). $20 \mu \mathrm{g}$ samples were separated by $8-12 \%$ SDS-PAGE, and then the protein was transferred to PVDF membranes $(0.45 \mu \mathrm{m}$ or $0.22 \mu \mathrm{m}$, Millipore, USA) and were blocked with $5 \%$ fat-free milk. After blocking for $2 \mathrm{~h}$, the membranes were washed three times for $10 \mathrm{~min}$ per time by TBST. The membranes were incubated with indicated primary antibodies : SNAPIN (10055-1-AP, proteintech, 1:500), phosphor-PKAC-a (D45D3, cell signaling technology,1:500), PKAC-a (D38C6, cell signaling technology,1:1000), PPP3CC (ab154863,abcam,1:1000), P38 (phosphorT180+Y182, ab195049, abcam, 1:500), P38 (ab47363, abcam, 1:1000), Bcl-2(ab194583, abcam, 1:3000), Bax (ab173026, abcam, 1;3000) and GAPDH (ab8245, abcam, 1:4000) overnight at $4^{\circ} \mathrm{C}$. Then the membranes incubated with primary antibodies goat anti-rabbit IgG or rabbit anti-mouse after washed three times for $2 \mathrm{~h}$. ChemiDoc ${ }^{\mathrm{TM}}$ MP Imaging system (Bio-Rad Co, USA) and ImageJ software were used to quantify the protein bands.

- 2.13 Molecular docking simulations

The conformation of proteins was downloaded from Protein Data Bank (PDB) database (www.rcsb.org/), and the structures of compounds were obtained from PubChem and Chem3D 18.0. The proteins of PDB format and compounds were final saved as PDBQT format after removing water, adding hydrogens, computing gasteiger, assigning AD4 type, detecting the root, choosing Torsions. MGLTools 1.5.6 
(www.mgltools.scripps.edu/) was use to operate above operations. Auto Dock Vina 1.1.2 (www.vina.scripps.edu/) was used to figure out the docking scores of binding affinities about targets and corresponding compounds (more negative scores means higher binding affinities). The molecular docking results were visualized by the Pymol Molecular Graphics System (Version 2.3.0)

- 2.14 Statistical analysis

For comparisons between two groups, we used Student's unpaired t-test. One-way ANOVA with post-hoc test was applied to determined significant differences for multiple groups. All data are shown as mean \pm $\mathrm{SE}$, and $\mathrm{p} \leq 0.05$ was considered.

\section{Results}

\subsection{HPLC analysis identified constituents of CuraUltra}

The chemical composition of CuraUltra was identified by UPLC-Q-Orbitrap MS, and accurate mass measurement of each molecular ion peak was carried out in positive and negative ion modes. The major components were well separated and detected under optimized UHPLC and MS conditions (Figure. 1A and B). Import the data into Thermo Xcalibur Qual Browse software, on the basis of summarizing the chemical composition and cleavage rules of the single medicine in the CuraUltra compound. The relative molecular mass of the compounds was analyzed according to the molecular ion peak of the positive and negative ion mode, and the element composition was calculated. And then the compounds were qualitatively identified by comparing chromatographic retention time and MS/MS information. Finally, 38 compounds were identified from CuraUltra including such as Gastrodin, SibiricoseA5, 3, 6'-Disinapoly sucrose, OnjisaponinB, Echinacoside, Acteoside, Curcumin, 1', 2'-dihydroxyasarone (Supplementary Table 1).

\subsection{CuraUltra ameliorated scopolamine-induced learning and memory deficit}

To evaluate the improvement of learning, memory ability, and spatial cognition, novel object recognition (NOR) test, step-down passive avoidance (SDA) test, and Morris water maze (MWZ) test maze tests were conducted (Figure 2). In the NOR test, scopolamine-treated group (model group) exhibited low-level features in recognize recognition index compared with control group, in contrast, CuraUltra or HupA treatment significantly improved the reduced RI in AD mice (Fig. 2A). By SDA test, we detected that the treatment of CuraUltra notably recovered scopolamine-induced shorter step-down latency (Fig. 2B) and more errors (Fig. $2 \mathrm{C}$ ) of $A D$ mice. In the Morris water maze (MWZ) test, the AD mice showed learning and memory deficits than that of control group, while CuraUltra improved cognitive impairment shown by the decreased escape latency to find the platform site and more crossing numbers in platform area on the final day after removed the platform. During the exploration period, decreased time of staying at target quadrant was also observed in scopolamine treated mice (Fig. 2D and E). Additionally, the escape latency recorded during positioning navigation test showed that $A D$ mice took longer to reach the platform 
(escape latency) than that of the control and CuraUltra group, with the effect most prominent on Day 4 (Fig. 2F), indicating significant spatial learning and memory impairment following scopolamine exposure.

\subsection{CuraUltra ameliorated scopolamine-induced hippocampal neuron damage}

First, Nissl staining was used to evaluate the pathological changes in hippocampus. In the AD group, the number of Nissl bodies in hippocampal neurons was reduced with a shallow staining. however, increased number of Nissl bodies and typical deep staining were observed in the CuraUltra group, compared with model group. To further evaluate the neuroprotective of CuraUltra on hippocampal neuron damage, cholinergic damage as indicated by decreased Ach level and increased AchE activity was evaluated, which was proved to be essential for the synaptic plasticity and normal hippocampal function. As shown in Figure. 3B and3C, compared with control group, scopolamine-treated model mice induced a remarkable decrease of Ach content and further a significant increase of AchE activity in the hippocampus of brain. In contrast, the CuraUltra or HupA treatment group significantly reversed the cholinergic alterations, as shown by the elevated AchE activity, confirming an ameliorating effect of CuraUltra on cholinergic function after scopolamine exposure.

Since apoptosis has been reported to be related to mechanisms of central cholinergic system dysfunction, and apoptosis damage induced by the cholinergic system abnormalities might be one main reason of scopolamine-induced cognitive decline, thus, we next sought to examined the expression levels of anti-apoptotic protein $\mathrm{Bcl}-2$, pro-apoptotic protein $\mathrm{Bax}$ by western blotting, and observed that comparing with control animals, obviously higher expression of Bax (Figure. 3D) and a decreased protein expression of $\mathrm{BCl}-2$ (Figure. $3 \mathrm{E}$ ) in scopolamine-treated mice had been found. Whereas CuraUltra treatment attenuated the scopolamine-mediated increase in Bax protein expression and reduction in $\mathrm{Bcl}-2$ expression in the brain.

\subsection{General features of the proteome}

We performed quantitative proteomic analysis using three replicates of hippocampal proteomes obtained from scopolamine-induced AD mice, the workflow chart was shown in (Fig. 4A). Across the treatments, proteins that exhibited significant quantitative difference among groups (ratio fold change $>1.2$ or < $0.883, p<0.05$ ) were defined as differential expression proteins. Changes in protein levels were shown in (Fig. 4B). The number of identified peptides and proteins were 42613 and 5402, respectively. A total of 4112 quantified proteins were identified (unique peptides $\geq 2$ ) (Supplementary Table 2). The overlaps of differentially expressed proteins between mod vs. control and CuraUltra vs. mod were 19 (Fig. 4C). Among these, 5 proteins affected by the CuraUltra treatment showed a callback trend, and they were found through literature review that they were closely related to AD. The volcano plot was performed to visualize the differential expression of identified proteins between CuraUltra and model group (Fig. 4D).

\subsection{Quality assessment of the proteomic data}


First, to verify whether the hippocampal proteome data of AD mouse model could be applied to further AD studies, we assessed the quantitative reproducibility (differences between technology and experimental settings) of the hippocampal proteomes. Log 2ratio distribution in each group (Fig. 5A) shown that there was no significant quantitation variation between the values of the combined sample channels from each group, suggesting that the distribution of ratiometric data was almost normal.

The relative standard deviation (RSD) values (Fig. 5B) varied from 0.114 to 0.161 , the SD of RSD values in each group ranged from 0.126 to 0.171 . The overall RSD values exhibit a narrow range of variability between different quantitative proteins among each group. Thus, the high degree of quantitative accuracy offered by the label-free quantification (LFQ) technique allows us to investigate proteomic alteration in the pathological development of $A D$.

Next, the hierarchical clustering present clear separation of the samples between model and CuraUltra groups (Fig. 5C), implying that protein expression across the samples undergoes extensive changes during $A D$ pathogenesis. In summary, it can be considered that the observed differences in quantitative protein expression reflect the diversity of molecular changes between groups in mouse tissues, rather than the effect of our label-free strategy. Thus, the workflow for proteomic analysis was stable and reliable, and could be used for the next mechanism evaluation.

To explore the mechanism of action of CuraUltra against $A D$, we next examined the biological function and pathways altered in mouse model. The Biological process of Gene Ontology (GO) analysis indicated that these differentially expressed proteins were mainly enriched in the positive regulation of autophagosome, presynaptic active zone membrane, ATPase activator activity, postsynaptic potential, postsynapse assembly, consistent with the fact that the pathology of $A D$ correlate positively with synaptic function in hippocampal neurons (Fig. 6A). KEGG analysis revealed significant pathways with $p$ $<0.05$ (Figure 6, CuraUltra group vs. Model group). For the KEGG pathway analysis, the highly enriched terms included Ribosome, Glutamatergic synapse, Pyrimidine metabolism, Dopaminergic synapse, Tight junction, Toxoplasmosis, Endocytosis, RNA transport, Spliceosome (Fig. 6B). These results demonstrate that the modifications observed in synaptic function may contribute to the beneficial effects of CuraUltra treatment in AD.

To further select the hub altered proteins closely related to disease and speculate the probable interactions of the differentially expressed proteins, 214 differential proteins enriched in the model group vs. CuraUltra group were submitted to the string database. The network analysis based on protein-protein interaction (PPI) showed that several of the altered proteins were significantly connected with each other (FDR $<0.05$; enrichment value $p<0.001$ ) and most of the related differential proteins were mainly concentrated in five sub-network clusters: Nervous system development, Regulation of ATPase activity, Neuron projection development, Ribosome and Glutamatergic synapse, many of which were related to neurological function, contributing to AD pathology. Meanwhile, the predictive biological function and pathways obtained from network model are similar to GO and KEGG, which further explains that they are significantly altered in model mice (Fig. 7). 
To examine the specific protein changes under the sub-network clusters that related to AD pathologies, LFQ intensity of each sample was investigated, the 'protein expression level' was represented by the ratio of individual samples compared to pooled samples, named "normalized protein abundance". As shown in (Fig. 8A), proteins involved in glutamatergic synapse signaling pathway such as Serine/threonine-protein phosphatase (PPP3CC), GTPase-activity related proteins Ras GTPase-activating protein 4(RASA4), DnaJ homolog subfamily B member 1 (DNAJB1), and neuron projection development related-proteins SNAREassociated protein (SNAPIN), Tyrosine-protein kinase (MERTK) were significantly recovered after the administration of the CuraUltra treatment. This analysis revealed that some differentially expressed protein are altered at the 'protein level' in the hippocampus of AD mouse model following the CuraUltra treatment. The relevant sub-networks involved in glutamatergic synaptic pathways obtained from the KEGG pathway database were depicted in (Fig. 8B). Notably, the PPP3CC (also known as calcineurin/PP2B) [18], cAMP-dependent protein kinase (PKA), and mitogen-activated protein kinase(P38MAPK) comprise in a common signaling module in the KEGG regulation, in agreement with previous mechanistic reports regarding the connections between these proteins. Eventually, PPP3CC, SNAPIN, PKA and P38MAPK were selected as further analysis target due to their closely relevance to neuronal synaptic plasticity.

\subsection{Validation of the protein targets}

To verify the differentially expressed proteins screened by proteomics, we measure the altered concentrations of PPP3CC, SNAPIN, P38MAPK, PKA proteins through qPCR and Western blotting. As shown in Figure 8, Comparing with controls, the downregulated expression of PPP3CC, SNAPIN, PKA (Fig. 9A, B, C) was observed in the hippocampus of mice after exposure to scopolamine, as well as an elevated P38MAPK expression (Fig. 9D) ( $\mathrm{p}=<0.005)$, however, scopolamine-induced the decreased of PP3CC, SNAPIN, PKA expression and activation of P38MAPK in the hippocampal was ameliorated by the treatment with CuraUltra. Consistent with the data obtained from western blotting analysis, we also observed an elevated level of SNAPIN and PPP3CCmRNA expression (Fig. 9E, F) in the scopolaminetreated model mice as compared with the control mice. RT-qPCR and Western blot results were consistent with those of the proteomic analysis.

\subsection{Molecular Docking}

To verify the interaction between the traditional Chinese medicine compound active compound and the key targets screened by proteomics, The 38 main components contained in the CuraUltra formulation were docked with PPP3CC, SNAPIN, PKA, and P38MAPK using AutoDock Vina. The Vina score (affinity $(\mathrm{kcal} / \mathrm{mol}))$ indicates the binding activity between the receptor and ligand. The more negative the value of binding free energy, the more stable between ligand and receptor [19]. Most of the compounds had good binding activity with PPP3CC, SNAPIN, PKA and P38MAPK (Supplementary Table 3). Using pymol, the highest affinity toward compound-target pair was employed as an example for visualization (Fig. 10).

\section{Discussion}


The use of TCM as a potential treatment strategy for AD is an evolving field of investigation. However, there is still a lack of strong experimental evidence and mechanism elaboration of TCM treatment. More effective therapy is under investigation. In the present study, we investigated the protective effects of CuraUltra in attenuating cognitive impairment evoked by scopolamine. Scopolamine exposure in mice has been demonstrated previously to induce cholinergic decline and apoptosis in brain, together with cognitive decline [20], which is considered as "scopolamine dementia" [21]. As expected, we found that CuraUltra treatment significantly ameliorate cholinergic dysfunction and neuronal apoptosis by improving Ach content and AchE activity, and regulating the expression of apoptosis-related proteins (such as $B a x$ and $B c / 2$ ), thereby exert nerve damage protective effects. In addition, the cognitive dysfunction in spatial learning and memory abilities were also observed in the hippocampus of scopolamine-triggered mice, as assessed by behavioral tests. Meanwhile, the level of hippocampal neuron damage was improved by CuraUltra compared with scopolamine group. Apart from the analysis of protective efficacy of CuraUltra in improving cognitive impairment. Acute oral toxicity and 13-week subacute toxicity studies were conducted. Safety evaluations on clinical observation, body weight, hematology, histopathological examination, ophthalmological examination did not show adverse changes. Together with previous work, the present investigation has established that the administration of CuraUltra is well effective and safe.

To investigate the therapeutic targets of CuraUltra and its mechanism of action for AD treatment, we employed in-depth quantitative proteomics to characterize the abnormal alterations of the hippocampal proteomes obtained from scopolamine-induced AD mice. In total, there were 252 proteins were revealed to be differentially expressed following CuraUltra treatment. Next, we sought to investigate the biological pathways altered by differential proteins in $A D$ mouse model to gain new insight into the pathogenesis of AD. Our data from bioinformatic analysis shown that many of the disease-associated differentially expressed proteins are mainly involved in synaptic function-associated processes such as postsynaptic potential, postsynapse assembly, glutamatergic synaptic pathway. Among the pathways related to the nervous system, some proteins belonging to the glutamatergic synapse signaling pathway, forming an interaction bridge to promote synaptic homeostasis, such as PPP3CC, PKA and MAPK. Furthermore, western blot and qPCR analysis clearly confirmed the expression levels of these proteins in hippocampus of scopolamine-induced model mice, providing a mechanistic basis for the synaptic dysfunction and cognitive impairment seen in AD.

The hippocampus is the main brain area required for the forms of learning and memory, and synaptic plasticity has long been described as a key component of learning and memory. And, the process can induce by the activation of N-methyl-d-aspartate receptor (NMDAR) [20]. Consistent with it,PPP3CC, which encodes a catalytic subunit of calcineurin ( $\mathrm{CaN}$; also named protein phosphatase 2B, PP2B) [23], exhibit an extremely intimate relation with NMDAR and synaptic development in hippocampal neurons. It was proved that calcineurin could regulate glutamatergic transmission by indirectly influencing NMDARs [24], and the observed increase in NMDAR might be attributable to the increased levels of PPP3CC [25]. Also, a reduction in PPP3CCmRNA levels was observed in the hippocampus neurons under pathological conditions in vivo [26], such increases in expression contribute to the dysfunction of synaptic plasticity 
leading to schizophrenia pathogenesis. Taken together, these data provide evidence that PPP3CC might serve as a signal transducer for synaptic enhancement and memory formation in the hippocampus. Notably, however, the role of $P P P 3 C C$ in AD has not been fully described previously. We show here for the first time in vivo the pivotal role of PPP3CC played in AD. As observed in scopolamine-induced mice, functionally, administration of CuraUltra increased the expression of PPP3CC in the hippocampus, adding to evidence PPP3CC implicating pathological process of AD. Additionally, protein kinase $A(P K A)$ has also been proven to be a central regulator of neuronal synaptic plasticity. A previous study identified that $P K A$ activation is able to alleviate learning and memory deficits stemmed from $A \beta$ accumulation in an $A D$ mouse model, thereby further affects the progress of AD [27]. More importantly, several evidences suggest that there was a closely correlations between efficient increase of calcineurin and $P K A$ activity [28-29]. According to reports, calcineurin acts upstream of $P K A$, which is recognized to affect the latter by augmenting the $P K A$ level [30]. Also, observations from in vitro cultured neurons prepared from calcineurin-/- mice proposed that some forms of synaptic plasticity-related abnormalities may be associated with loss of calcineurin-mediated cAMP increases [31]. Given the potential association between $P P P 3 C C$ and $P K A$, which coordinate synaptic plasticity function, may be an important molecular mechanism in $A D$ treatment, we next investigated whether the decreased phospho-PKA in mouse model could be altered following CuraUltra treatment. By Western blotting and qPCR analysis, we revealed a significant elevation of $P P P 3 C C$ protein level in scopolamine mice, accompanied by an increased $P K A$ activity but not in the model. It appears that augmenting PPP3CC levels may more likely to lead to increased phosphorylation of PKA expression, further, leading to the preservation of spatial learning/memory and protection against synaptic dysfunction. Additionally, administration of CuraUltra also reverse the up-regulation expression of P38MAPK. The mitogen-activated protein kinase (MAPK) family comprises p38 proteins, ERK1/2 and JNK [32]. Numerous studies have demonstrated that activated $P 38 M A P K$ has been found in human $A D$ brain tissue, furthermore, recent studies have corroborated the fact that there is an interaction between phospho-PKA and phospho-P38MAPK expression. In most systems, MAPK stimulates the transcriptional activity of NF-kB, while $P K A$ suppresses it [33]. In addition, PKA activator-SKF38393 was in fact developed as potential therapeutics for AD with purpose to block P38MAPK phosphorylation[34]. It seems likely that the increased PKA phosphorylation level after CuraUltra treatment can not only be the consequence of the activated PPP3CC but also contribute to the inhibition of $p 38$ MAPK phosphorylation. From the above, we reasoned that the activation of $P P P 3 C C$ and $P P P 3 C C$-regulated $P K A$, acting synergically in the suppression of $P 38 M A P K$, forms a reinforcing cycle required to support homeostatic synaptic plasticity at the level of the glutamatergic synapse pathways, which might result in the strengthening of specific neuronal networks in the brain. Taken together, these results support the conclusion that the neuroprotective effect of CuraUltra treatment against AD may be through modulation of glutamatergic synapse cascade extrapolated from bioinformatics analysis.

In addition to PPP3CC, the SNAPIN related to presynaptic regulation was also a novel indicator involved in the differential proteins we screened. SNAPIN, which is a SNARE-associated protein, was abundantly expressed in neuronal cells. A study of Pan et al. proposed the potential of SNAPIN as a functional target 
for modifications of cognitive disorders, they also proved that in vitro deficiency of SNAPIN in brain neurons from snapin-deficient mice impairs the precision and efficacy of synaptic transmission [35]. Our results further provided information about the protein and gene expression of SNAPIN in the hippocampus of AD mice, highlighting the important role of SNAPIN reduction in the pathological process of $A D$. However, its mechanism to prevent nerve damage needs further investigation. Finally, Molecular docking revealed that these chemical constituents of CuraUltra show high binding activity towards the major target protein. Collectively, our results provide preliminary evidence for the potential of PPP $3 C C$ and $S N A P I N$ to serve as a novel therapeutic target for $A D$. There is no previous report suggesting that changes in the levels of PPP3CC and SNAPIN may be the pathological phenotype of AD brain. In addition, there are still few experimental studies aimed at elucidating the PPP3CC/PKA/P38MAPK axis during glutamatergic synapses. Therefore, our work provided novel insights into the molecular mechanism of synaptic plasticity alterations in the pathological process of $A D$, and further confirmed the effectiveness of CuraUltra as a natural herbal formula to regulate synaptic strength. However, the present study also has limitations. More experiments need to be proceeded in the future to demonstrate the protective effect of CuraUltra for $A D$ treatment, and the mechanism of CuraUltra in mediating $A D$ by glutamatergic synapse pathway still need to be clarified.

In conclusion, the results of our study demonstrate that CuraUltra treatment can effectively alleviate scopolamine-induced cognitive impairment. The underlying mechanism may be related to the regulation of the PPP3CC-PKA-MAPK glutamatergic synapse pathway and SNAPIN expression.

\section{Abbreviations}




\begin{tabular}{ll} 
TCM & Traditional Chinese medicine \\
\hline AD & Alzheimer's disease \\
\hline UPLC-MS & ultra-performance liquid chromatography-mass Spectrometry \\
\hline LFQ & Label-free quantification \\
\hline RSD & Relative standard deviation \\
\hline PCA & Principal component analysis \\
\hline PPP3CC & Serine/threonine-protein phosphatase \\
\hline PKA & cAMP-dependent protein kinase \\
\hline SNAPIN & SNARE-associated protein \\
\hline P38MAPK & Mitogen-activated protein kinase \\
\hline Ach & Acetylcholine \\
\hline AchE & Acetylcholinesterase \\
\hline SDA & Step-down passive avoidance \\
\hline MWM & Morris water maze test \\
\hline NOR & Novel object recognition \\
\hline RASA4 & Ras GTPase-activating protein 4 \\
\hline DNAJB1 & DnaJ homolog subfamily B member 1 \\
\hline
\end{tabular}

\section{Declarations}

\section{Ethics approval and consent to participate}

The feeding procedures of animals and experimental operations have been approved by the Animal Care and Use Committee of Beijing University of Chinese Medicine (BUCM-4-2019102105-4119).

\section{Consent to publish}

Not applicable

\section{Availability of data and materials}

We analyzed publicly available datasets in the current study. These data can be found at: https://www.iprox.cn/page/project.html?id=IPX0003302000. 


\section{Competing interests}

There is no potential conflict of interest to disclose by authors.

\section{Funding}

This work was supported by grants from the medical security and capacity building of traditional Chinese medicine in 2020 (0332000000131).

\section{Authors' Contributions}

Qianqian Huang performed proteomics analysis, conducted experiments and drafted the manuscript. Yuanyuan Shi and Haibin Zhao conceived the research. Chen Zhang performed the experiments. Qihong Ma performed the UPLC/MS analysis. Shi Dong analyzed the data. Shanglong Wang and Zimin Liu performed compound preparation. All authors approved the submitted version.

\section{Acknowledgements}

In this section, we also thank Guohua Yu and Wenxiang Zhu for their scientific comments in writing this article, and thank Ying Hao for her guidance in proteomics analysis.

\section{References}

1. Liu, Y., et al., Short-term resistance exercise inhibits neuroinflammation and attenuates neuropathological changes in 3xTg Alzheimer's disease mice. Journal of neuroinflammation, 2020. 17(1): p. 4.

2. Lin Yaming, Chen Wei, Hu Linyuan, Duan Zhifang. Expert consensus on clinical application of Tianma Xingnao Capsule in the treatment of vascular cognitive dysfunction and neuropathic headache[J]. Chinese Journal of Traditional Chinese Medicine, 2021. 39(08): 255-258.

3. Qi Youli, Wang Lihua, Li Zhanxin.Progress in research on pharmacological effects and clinical application of Tianma Xingnao Capsule[J]. Chinese Medicines and Clinics, 2013. 13(10):1315-1316.

4. Chainoglou, E. and D. Hadjipavlou-Litina, Curcumin in Health and Diseases: Alzheimer's Disease and Curcumin Analogues, Derivatives, and Hybrids. International journal of molecular sciences, 2020. 21(6).

5. Li, M. and S. Qian, Gastrodin Protects Neural Progenitor Cells Against Amyloid $\beta$ (1-42)-Induced Neurotoxicity and Improves Hippocampal Neurogenesis in Amyloid $\beta$ (1-42)-Injected Mice. Journal of molecular neuroscience: MN, 2016. 60(1): p. 21-32. 
6. Xiao, M.-M., et al., Gastrodin protects against chronic inflammatory pain by inhibiting spinal synaptic potentiation. Scientific reports, 2016. 6: p. 37251.

7. She, G., et al., Absorbable phenylpropenoyl sucroses from Polygala tenuifolia. Molecules, 2011. 16(7): p. 5507-13.

8. $\quad \mathrm{Li}, \mathrm{G}$., et al., Onjisaponin B prevents cognitive impairment in a rat model of D-galactose-induced aging. Biomedicine \& pharmacotherapy = Biomedecine \& pharmacotherapie, 2018. 99: p. 113-120.

9. Dai, Y., et al., Echinacoside Suppresses Amyloidogenesis and Modulates F-actin Remodeling by Targeting the ER Stress Sensor PERK in a Mouse Model of Alzheimer's Disease. Frontiers in cell and developmental biology, 2020. 8: p. 593659.

10. Lin, J., et al., [Effect of acteoside on learning and memory impairment induced by scopolamine in mice]. Zhongguo Zhong yao za zhi = Zhongguo zhongyao zazhi = China journal of Chinese materia medica, 2012. 37(19): p. 2956-2959.

11. Panta, A., et al., Mir363-3p Treatment Attenuates Long-Term Cognitive Deficits Precipitated by an Ischemic Stroke in Middle-Aged Female Rats. Front Aging Neurosci, 2020. 12: p. 586362.

12. Wang, W., et al., Lentiviral-Mediated Overexpression of the $18 \mathrm{kDa}$ Translocator Protein (TSPO) in the Hippocampal Dentate Gyrus Ameliorates LPS-Induced Cognitive Impairment in Mice. Front Pharmacol, 2016. 7: p. 384.

13. Sun, B., et al., Effects of Vortioxetine on depression model rats and expression of BDNF and Trk B in hippocampus. Experimental and therapeutic medicine, 2020. 20(3): p. 2895-2902.

14. Chen, C., et al., Dauricine Attenuates Spatial Memory Impairment and Alzheimer-Like Pathologies by Enhancing Mitochondrial Function in a Mouse Model of Alzheimer's Disease. Frontiers in cell and developmental biology, 2020. 8: p. 624339.

15. Hao, Y., et al., Quantitative proteomics reveal the protective effects of EDS against osteoarthritis via attenuating inflammation and modulating immune response. Journal of ethnopharmacology, 2021. 271: p. 113780.

16. Zhang, L., et al., Tianlongkechuanling Inhibits Pulmonary Fibrosis Through Down-Regulation of Arginase-Ornithine Pathway. Frontiers in pharmacology, 2021. 12: p. 661129.

17. Zhang, W., et al., Proteomic analysis using isobaric tags for relative and absolute quantification technology reveals mechanisms of toxic effects of tris (1,3-dichloro-2-propyl) phosphate on RAW264.7 macrophage cells. Journal of applied toxicology : JAT, 2021.

18. Wigington, C.P., et al., Systematic Discovery of Short Linear Motifs Decodes Calcineurin Phosphatase Signaling. Molecular cell, 2020. 79(2). 
19. Ding, Q., et al., Elucidation of the Mechanism of Action of Ginseng Against Acute Lung Injury/Acute Respiratory Distress Syndrome by a Network Pharmacology-Based Strategy. Frontiers in pharmacology, 2020. 11: p. 611794.

20. Sohn, E., et al., Leaf Extract Improves Scopolamine-Induced Memory Impairment by Preventing Hippocampal Cholinergic Dysfunction and Neuronal Cell Death. International journal of molecular sciences, 2019. 20(14).

21. Wan, T., et al., FA-97, a New Synthetic Caffeic Acid Phenethyl Ester Derivative, Protects against Oxidative Stress-Mediated Neuronal Cell Apoptosis and Scopolamine-Induced Cognitive Impairment by Activating Nrf2/HO-1 Signaling. Oxidative medicine and cellular longevity, 2019. 2019: p. 8239642.

22. Lee, J.K. and N.-J. Kim, Recent Advances in the Inhibition of p38 MAPK as a Potential Strategy for the Treatment of Alzheimer's Disease. Molecules (Basel, Switzerland), 2017. 22(8).

23. Cottrell, J.R., et al., Calcineurin Ay is a Functional Phosphatase That Modulates Synaptic Vesicle Endocytosis. The Journal of biological chemistry, 2016. 291(4): p. 1948-1956.

24. Lin, L., et al., AKAP79/150 impacts intrinsic excitability of hippocampal neurons through phosphoregulation of A-type $\mathrm{K}+$ channel trafficking. The Journal of neuroscience : the official journal of the Society for Neuroscience, 2011. 31(4): p. 1323-1332.

25. Ambrozkiewicz, M.C., et al., The murine ortholog of Kaufman oculocerebrofacial syndrome protein Ube3b regulates synapse number by ubiquitinating Ppp3cc. Molecular psychiatry, 2020.

26. Eastwood, S.L., P.W.J. Burnet, and P.J. Harrison, Decreased hippocampal expression of the susceptibility gene PPP3CC and other calcineurin subunits in schizophrenia. Biological psychiatry, 2005. 57(7): p. 702-710.

27. Zhang, Z., et al., Activation of PKA/SIRT1 signaling pathway by photobiomodulation therapy reduces $A \beta$ levels in Alzheimer's disease models. Aging cell, 2020. 19(1): p. e13054.

28. Voss, M., et al., Calcineurin activity augments cAMP/PKA-dependent activation of V-ATPase in blowfly salivary glands. American journal of physiology. Cell physiology, 2010. 298(5): p. C1047-C1056.

29. Yang, $\mathrm{H}$., et al., Activation of cAMP-response element-binding protein is positively regulated by PKA and calcium-sensitive calcineurin and negatively by PKC in insect. Insect biochemistry and molecular biology, 2013. 43(11): p. 1028-1036.

30. Gomez, L.L., et al., Regulation of A-kinase anchoring protein 79/150-cAMP-dependent protein kinase postsynaptic targeting by NMDA receptor activation of calcineurin and remodeling of dendritic actin. The Journal of neuroscience : the official journal of the Society for Neuroscience, 2002. 22(16): $p$. 7027-7044. 
31. Chan, G.C.K., S. Tonegawa, and D.R. Storm, Hippocampal neurons express a calcineurin-activated adenylyl cyclase. The Journal of neuroscience : the official journal of the Society for Neuroscience, 2005. 25(43): p. 9913-9918.

32. Xu, Z., et al., The Role of ERK1/2 in the Development of Diabetic Cardiomyopathy. International journal of molecular sciences, 2016. 17(12).

33. Kim, J., et al., The flagellin-TLR5-Nox4 axis promotes the migration of smooth muscle cells in atherosclerosis. Experimental \& molecular medicine, 2019. 51(7).

34. Shao, X., et al., Dopamine 1 receptor activation protects mouse diabetic podocytes injury via regulating the PKA/NOX-5/p38 MAPK axis. Experimental cell research, 2020. 388(2): p. 111849.

35. Pan, P.-Y., J.-H. Tian, and Z.-H. Sheng, Snapin facilitates the synchronization of synaptic vesicle fusion. Neuron, 2009. 61(3): p. 412-424.

\section{Figures}


A

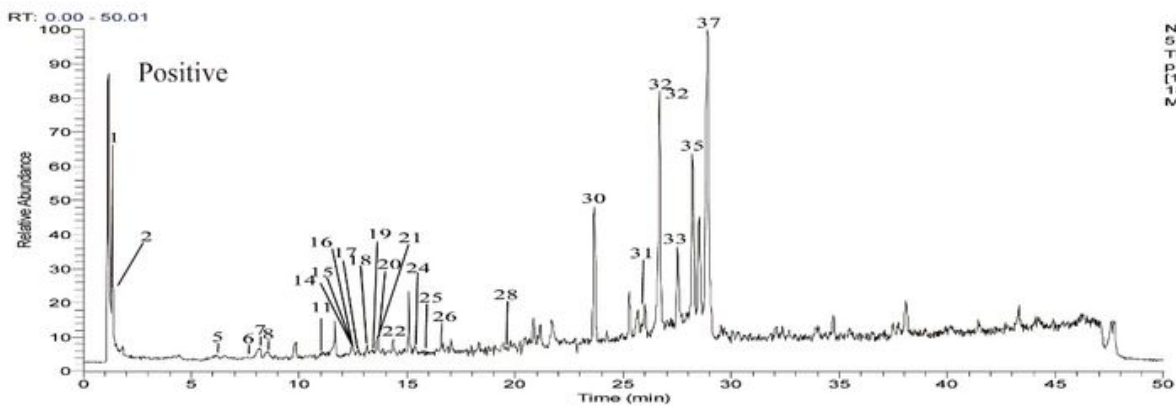

B

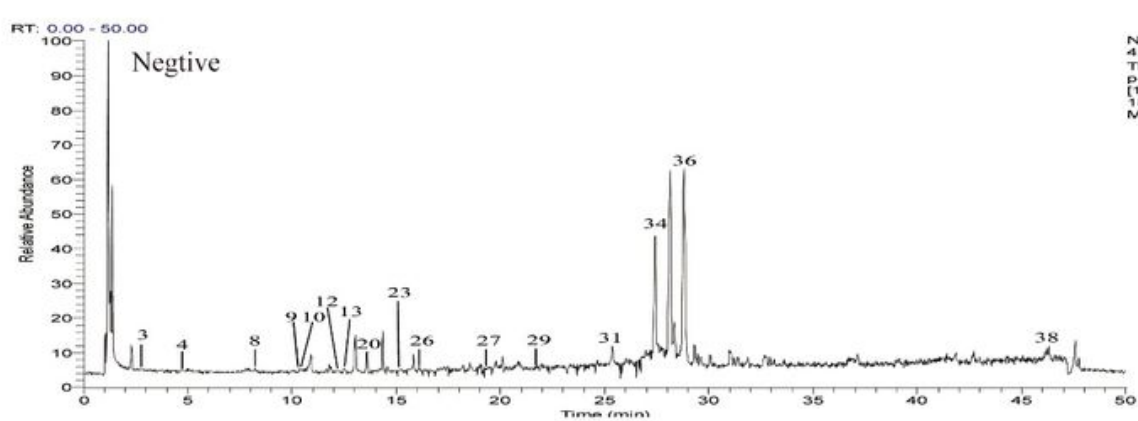

C
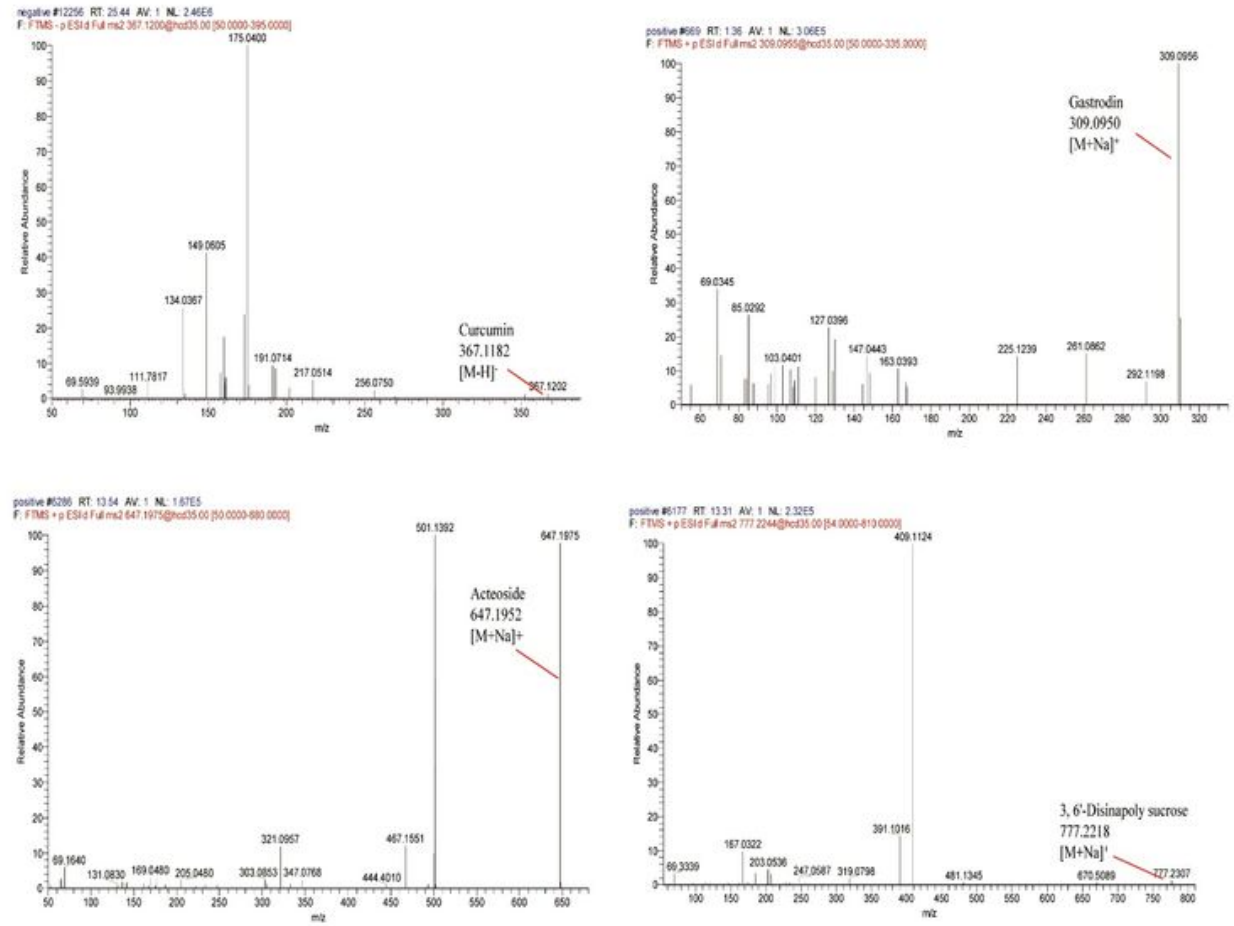

\section{Figure 1}

Total ion chromatogram monitored in positive (A) and negative (B) ion modes for CuraUltra; CuraUltra extract composition analysis: CuraUltra has Gastrodin, 3, 6'-Disinapoly sucrose, Echinacoside, Acteoside, Curcumin and other ingredients (C). 
A

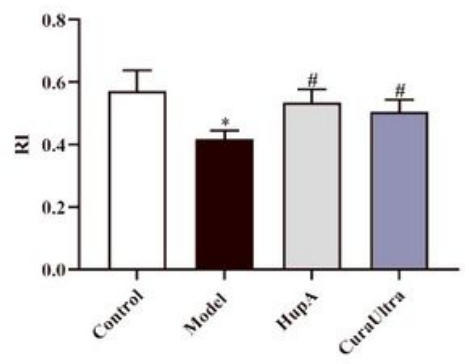

D

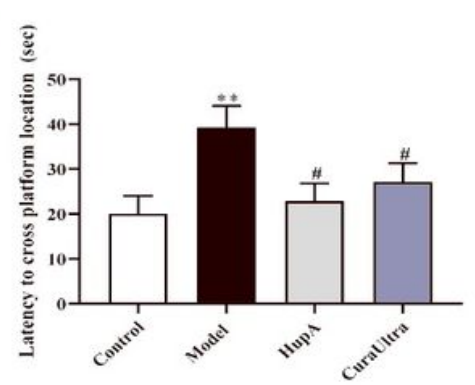

B
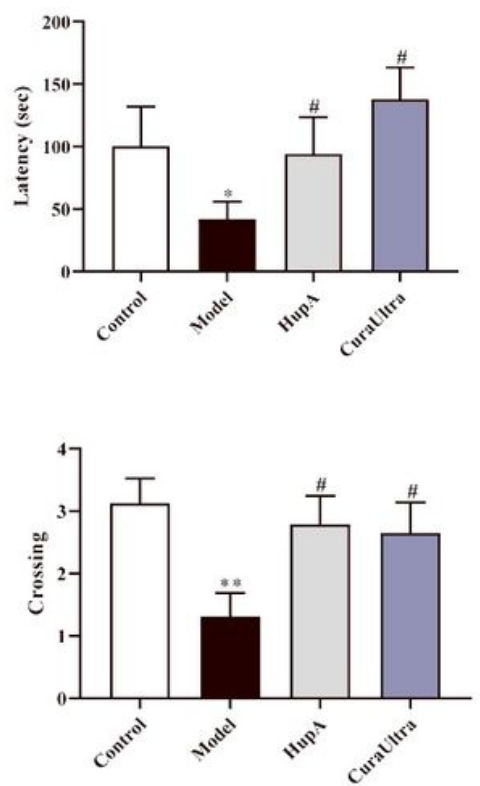
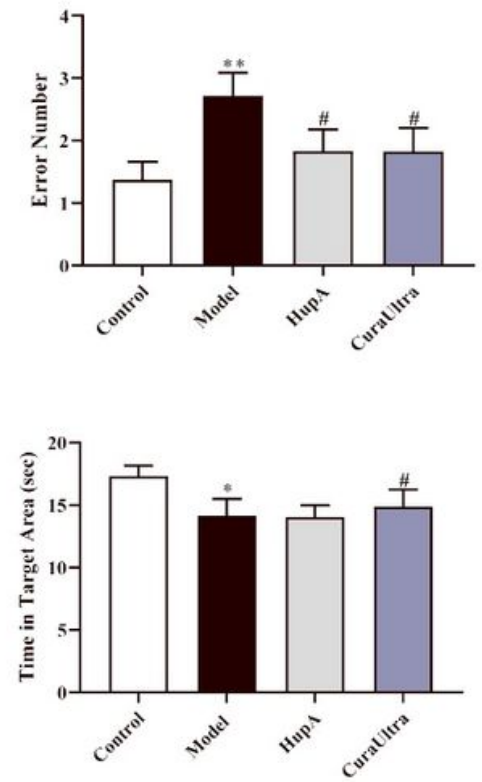

E
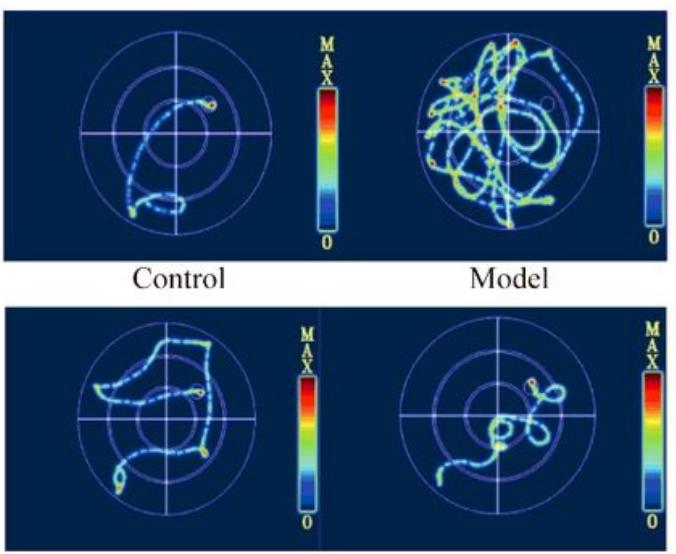

HupA

CuraUltra

C

F

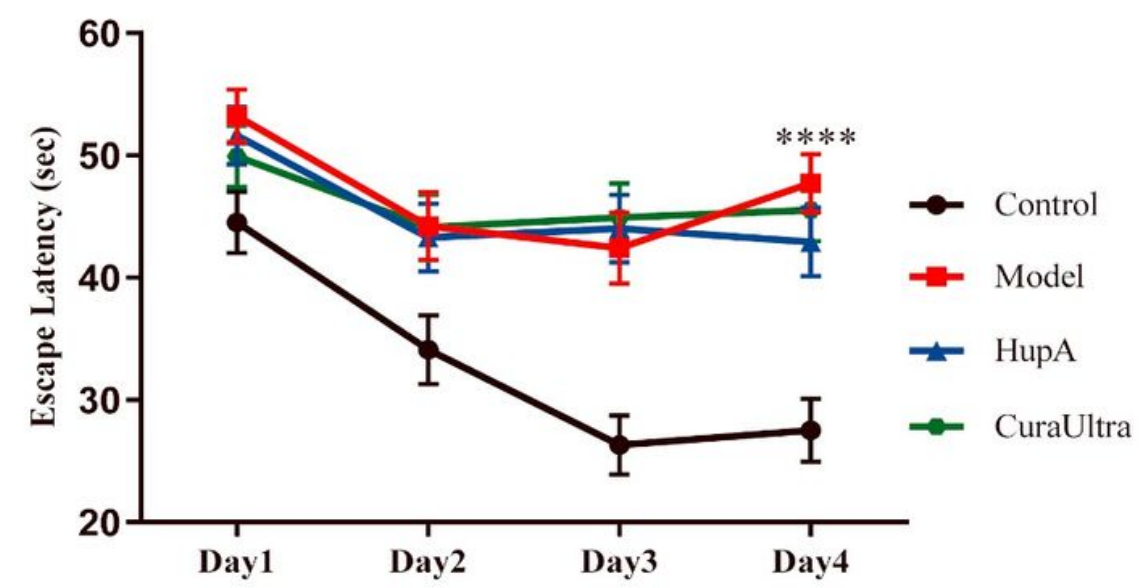

Figure 2

Ameliorating effect of CuraUltra on learning and memory deficits in scopolamine-treated mice. The exploration time percentage of novel objects (Recognition Index RI) in novel object recognition abilities $(A)$; The latency and the number of errors in step-down passive avoidance test $(B, C)$; The escape latency to find hidden platform, the numbers of crossing, retention time spent in target quadrant on the last day during Morris water maze (D); The representative swimming trace of the mice during memory test in the Morris water maze after removed the platform (E); The escape latency during positioning navigation test (the first 4 consecutive days training) (F). Data are expressed as mean \pm SE $(n=17)$. ${ }^{*} p<0.05,{ }^{*} p<0.01$ and ${ }^{* * *} p<0.001$ compared to the control group; \#p $<0.05$, \#\#p $<0.01$ and \#\#\#p $<0.001$ compared to the scopolamine-treated group. 


\section{A}

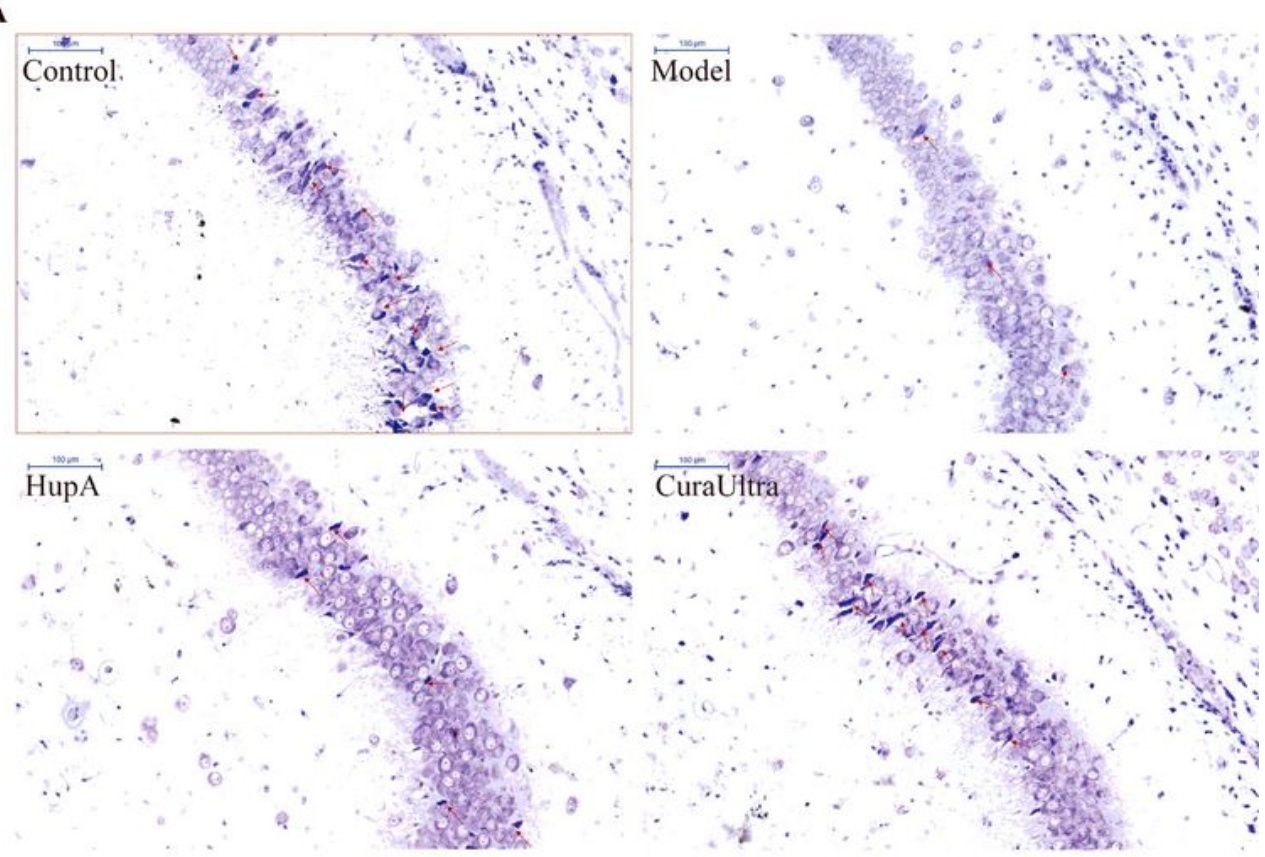

B

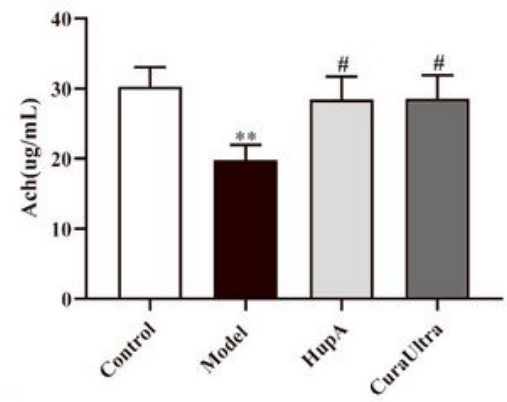

D
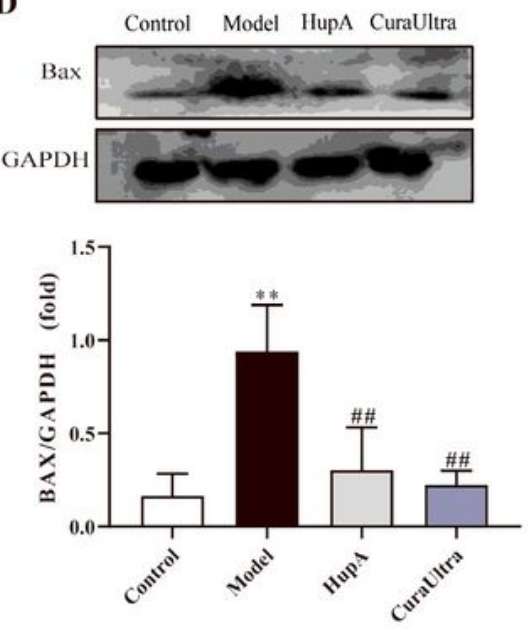

C

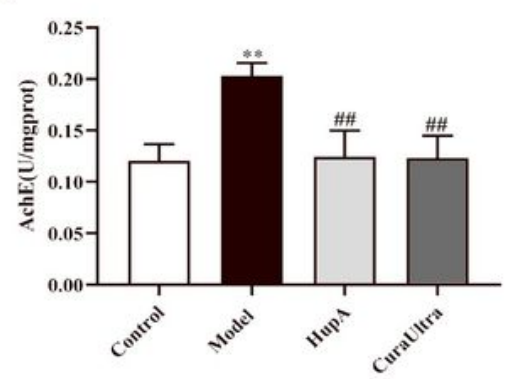

E
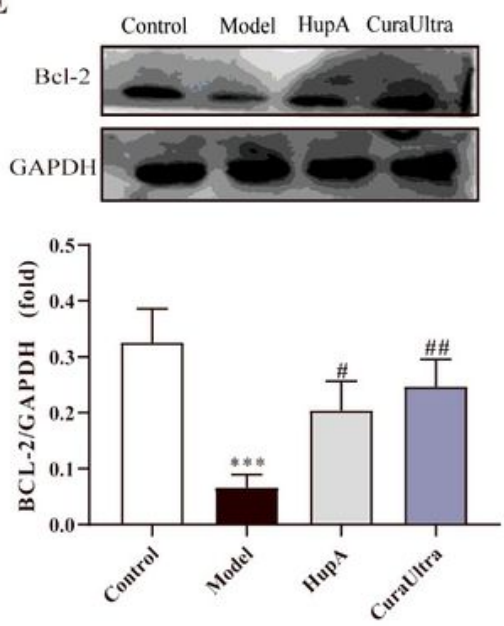

\section{Figure 3}

Ameliorating effect of CuraUltra on scopolamine-induced hippocampal neuron damage. Representative images of Nissl staining in hippocampal CA3 region(A). Ach content (B) and AchE activity (C) in hippocampus tissue were measured according to assay kit instructions; The expression of $\operatorname{Bax}(\mathrm{D})$ and bcl-2(E) in hippocampus tissue were detected by western blot $(n=3)$ and representative western blotting 
images; Data are expressed as mean \pm SE. ${ }^{*} p<0.05,{ }^{*} p<0.01$ and ${ }^{* \star *} p<0.001$ compared to the control group; \#p < 0.05, \#\#p < 0.01 and \#\#\#p < 0.001 compared to the scopolamine-treated group $(n=3)$.

A

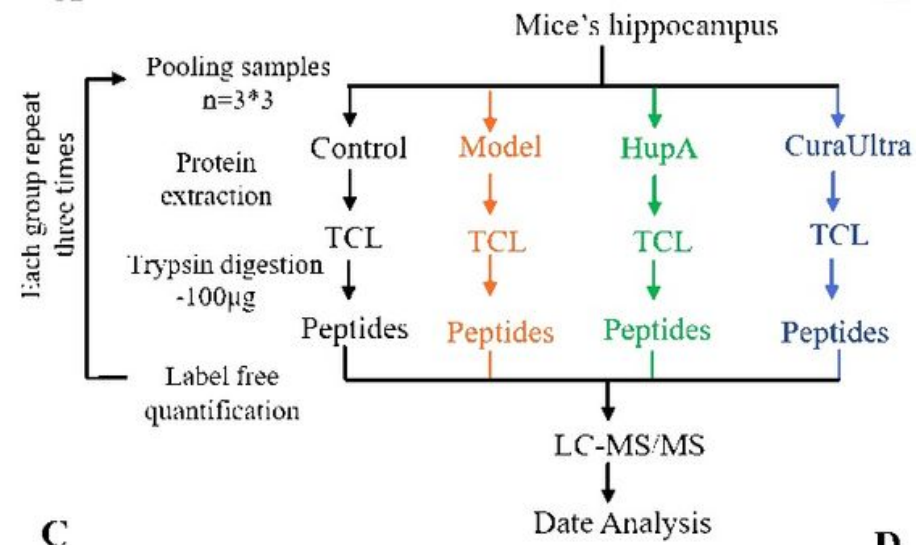

C.

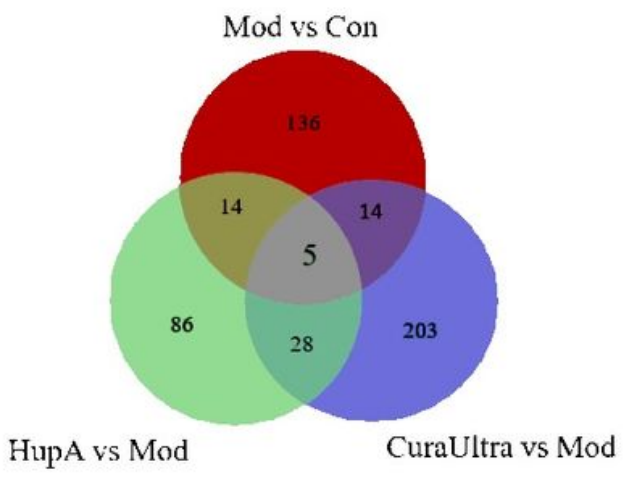

B

D

\begin{tabular}{cccc}
\hline & $\begin{array}{c}\text { Differentially } \\
\text { Expressed } \\
\text { proteins }\end{array}$ & $\begin{array}{c}\text { Up- } \\
\text { regulated }\end{array}$ & $\begin{array}{c}\text { Down- } \\
\text { regulated }\end{array}$ \\
\hline Mod vs Con & $136^{*}$ & $108^{*}$ & $28^{*}$ \\
Hup $\Lambda$ vs Mod & $86^{*}$ & $51^{*}$ & $35^{*}$ \\
$\begin{array}{c}\text { CuraUltra rs } \\
\text { Mod }\end{array}$ & $203^{*}$ & $128^{*}$ & $85^{*}$ \\
\hline
\end{tabular}

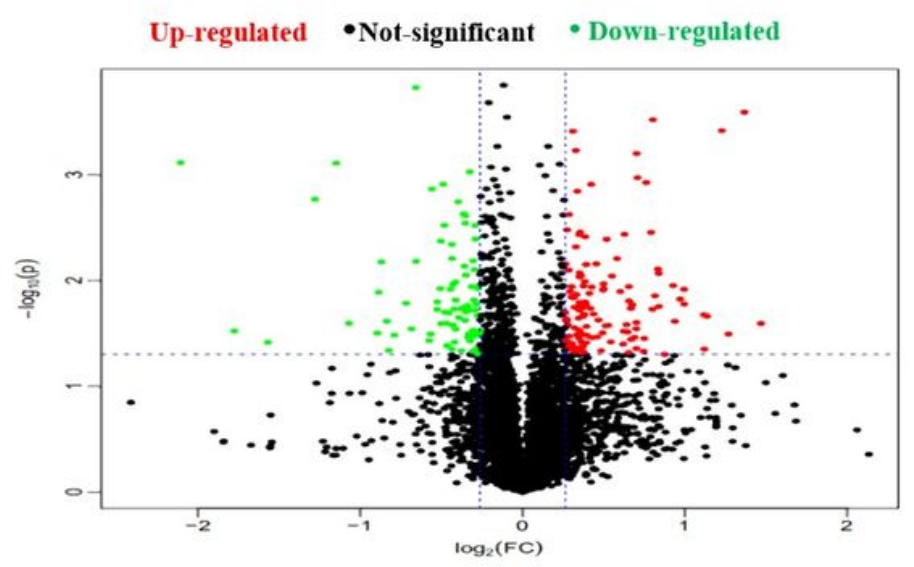

Figure 4

Evaluation of differentially expressed proteins. Graphical illustration of the workflow used for our LCMS/MS -based proteomic analysis (A); Identified up- or down-regulated proteins in hippocampal proteomics from each group (B); The cutoff values in the study set the fold change as $\geq 1.2$ or $\leq 0.883$ with $\mathrm{P}<0.05$ as the cut off value for statistical significance. Venn diagram analysis of differentially expressed proteins (C); Volcano map showing up- or downregulated proteins in a comparison of Mod and CuraUltra (D). Mod: scopolamine induced AD mouse. HupA: mod with huperzineA treatment mice. 
A

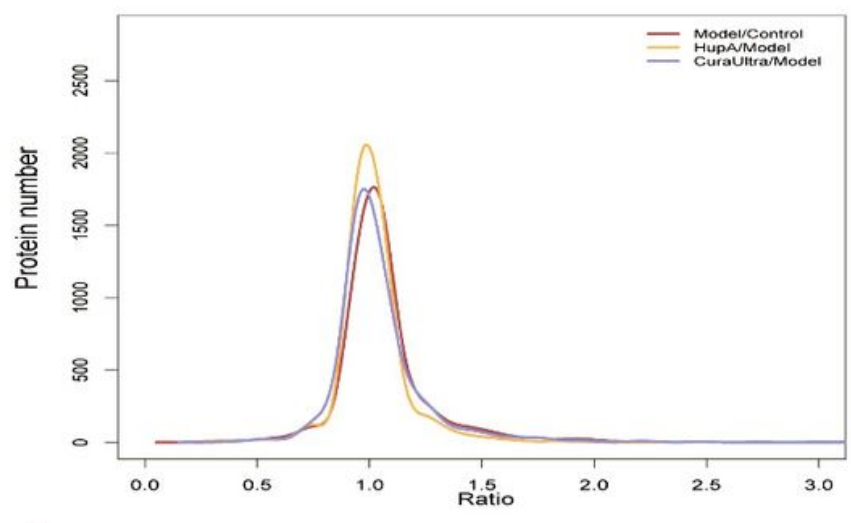

B
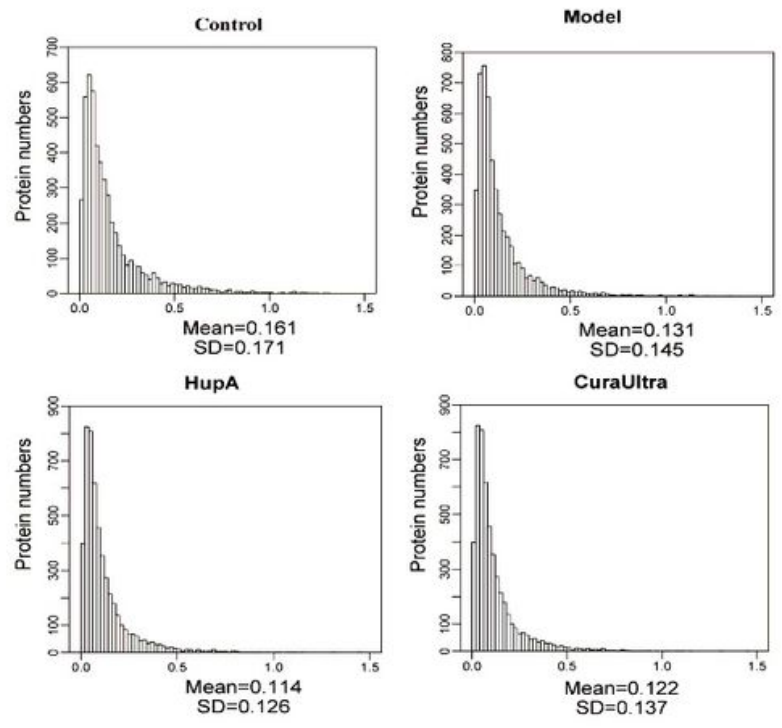

C

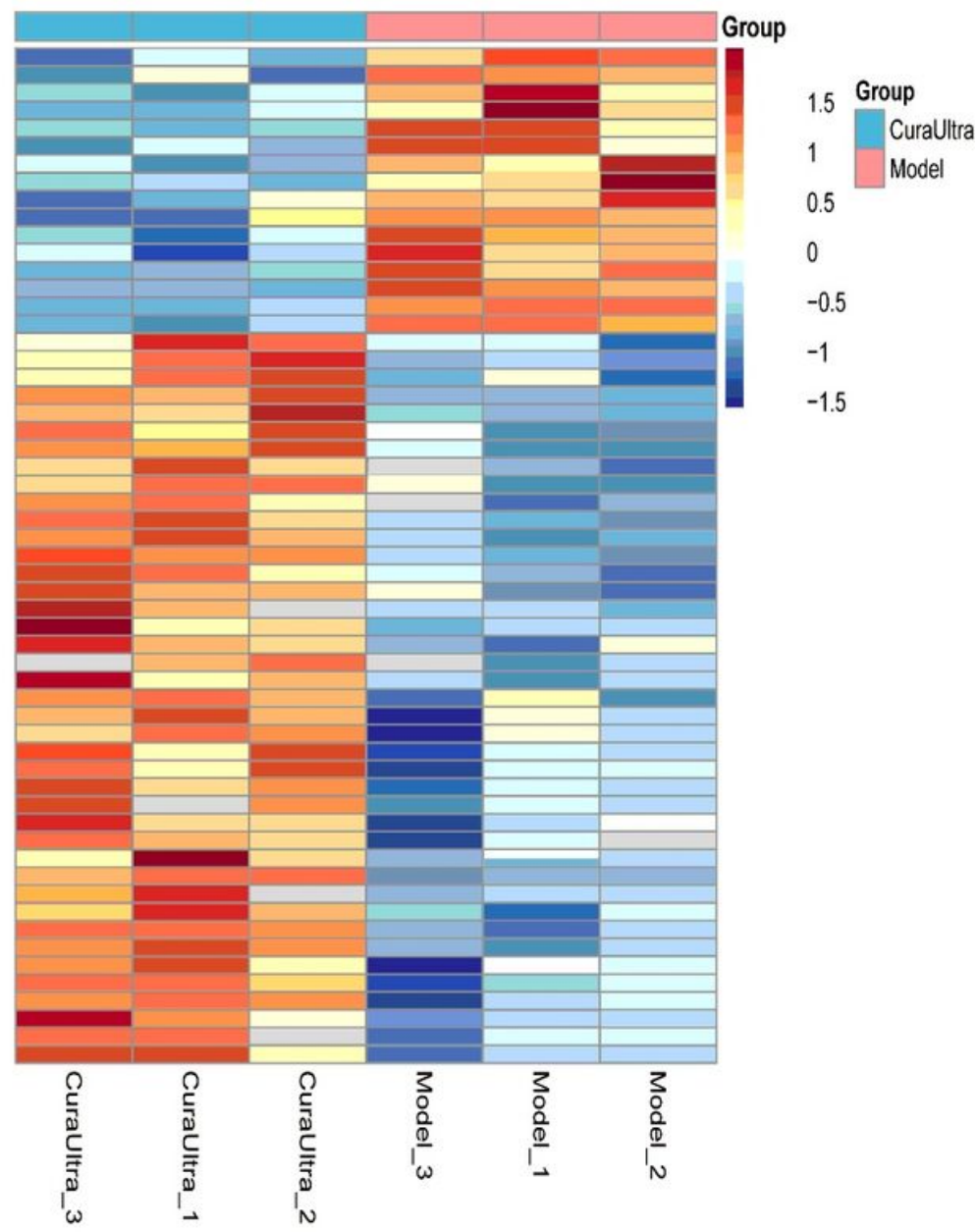

\section{Figure 5}

Quality assessment of the proteomic data. Log2ratio distribution of all differentially expression proteins detected in each group of three replicate samples, as determined by ANOVA based on FDR <0.05 (A); Distribution of relative standard deviation (RSD) in three pooled samples from each group (B); The hierarchical clustering analysis of hippocampal proteomes obtained from scopolamine-induced AD mice (C). 
A
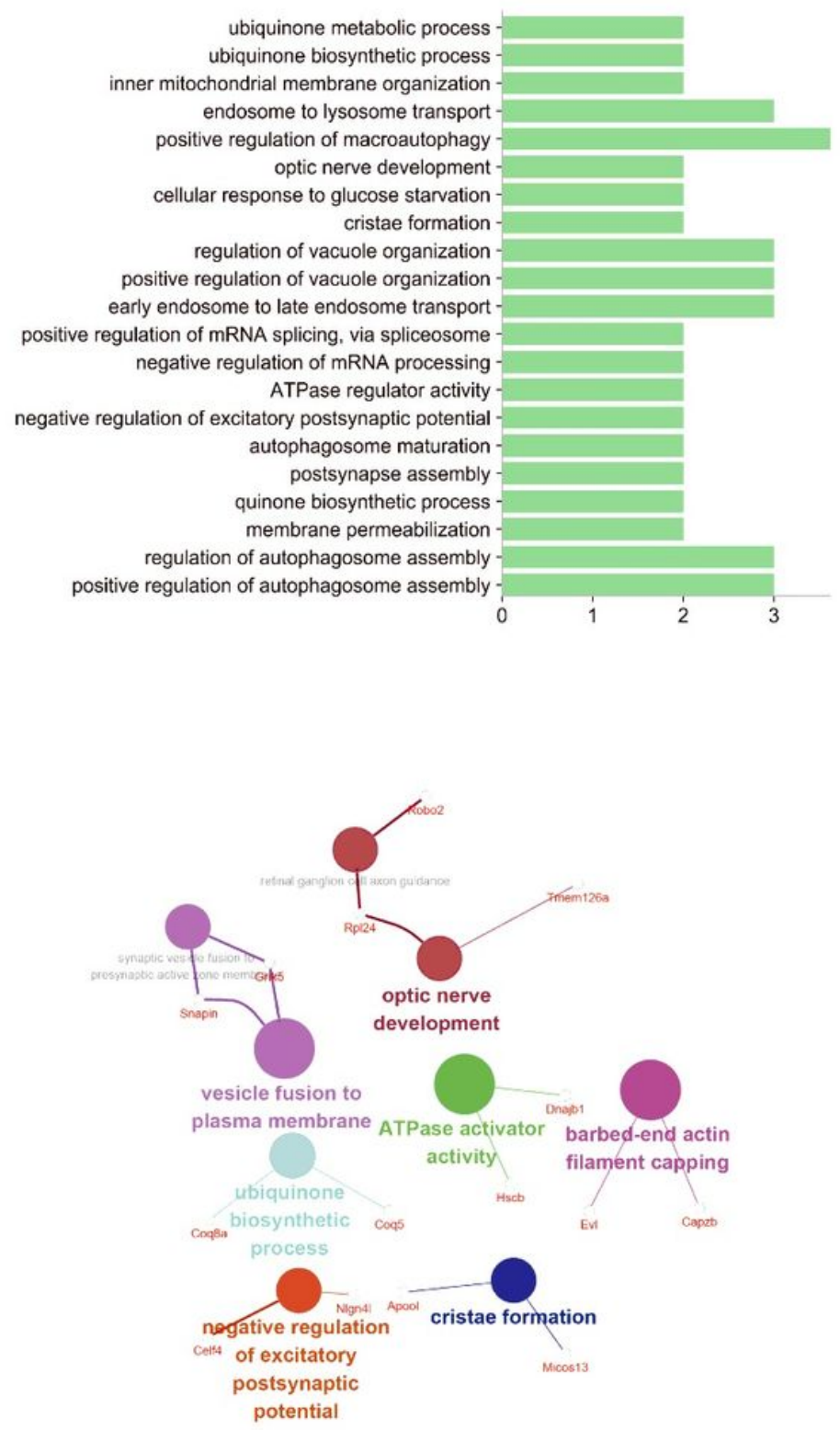

B
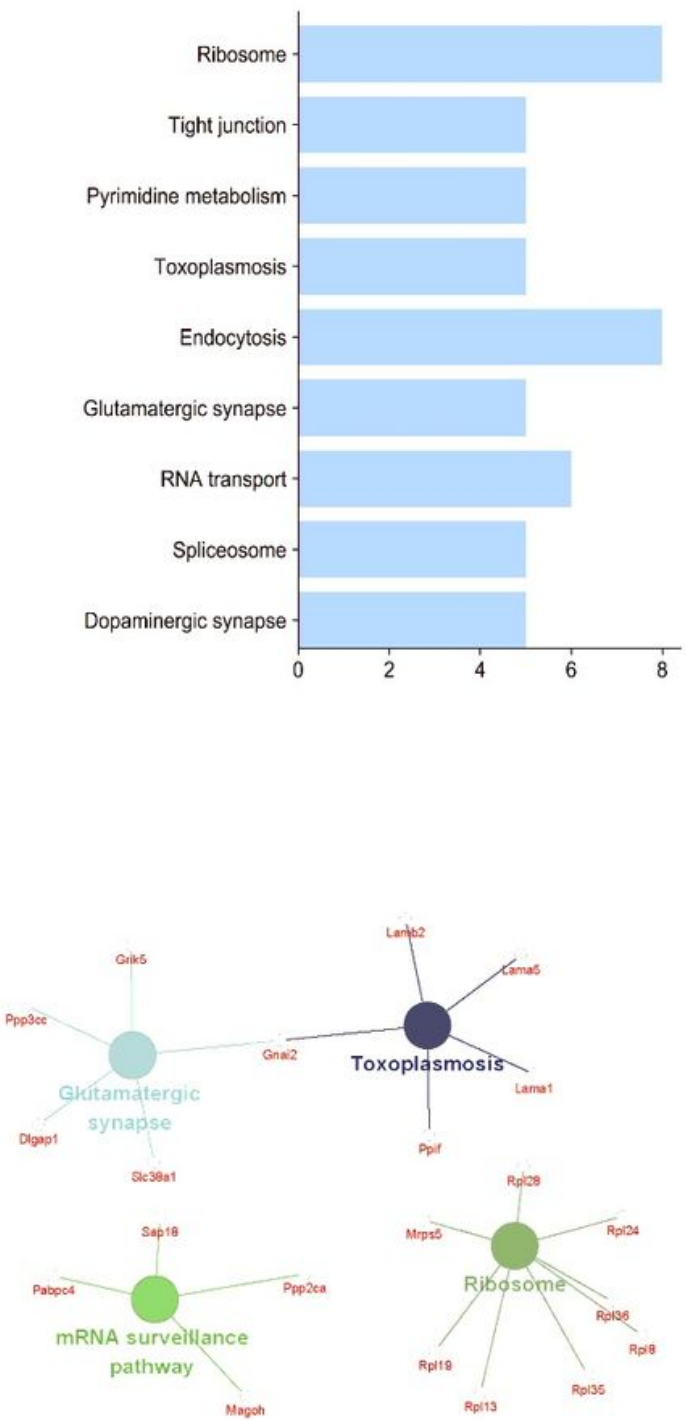

\section{Figure 6}

GO enrichment and pathway analysis of differentially expressed proteins along with their networking with associated proteins. The biological functions derived from the GO analysis (A); KEGG signaling pathway analysis for the differentially expressed proteins using Clue GO software and the p-value was set at $<0.05$ (B). 


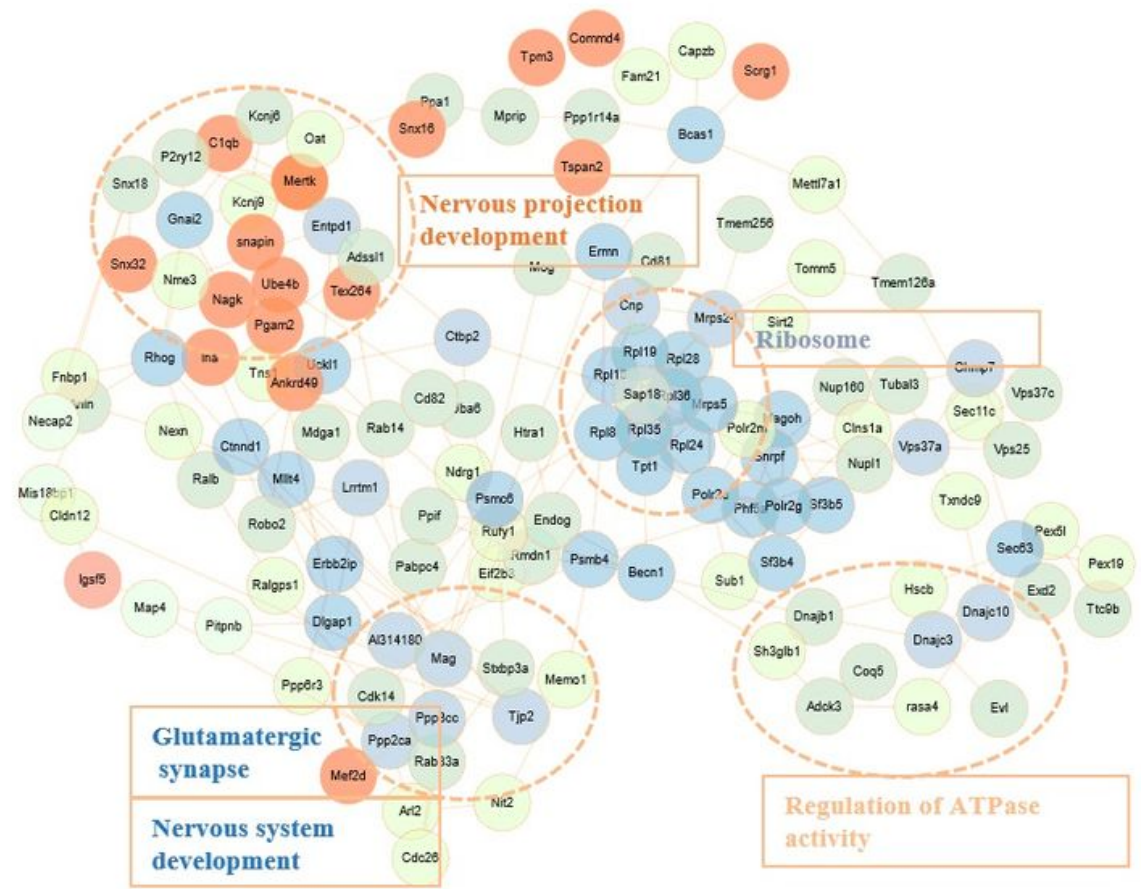

Figure 7

PPI network of potential targets. The network model was constructed to elucidate the functional proteinprotein interaction (PPI) of the 252 proteins between model and CuraUltra group. 
A
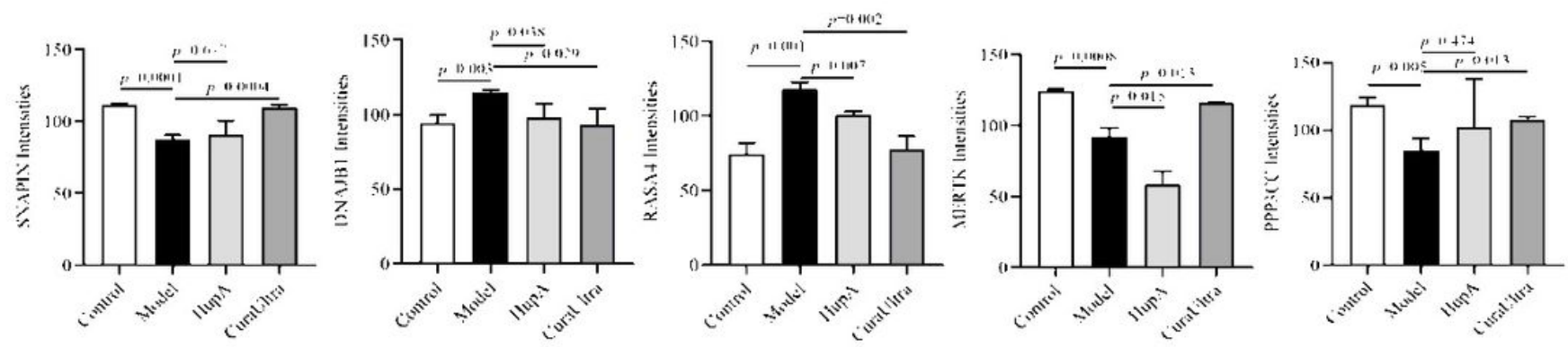

B

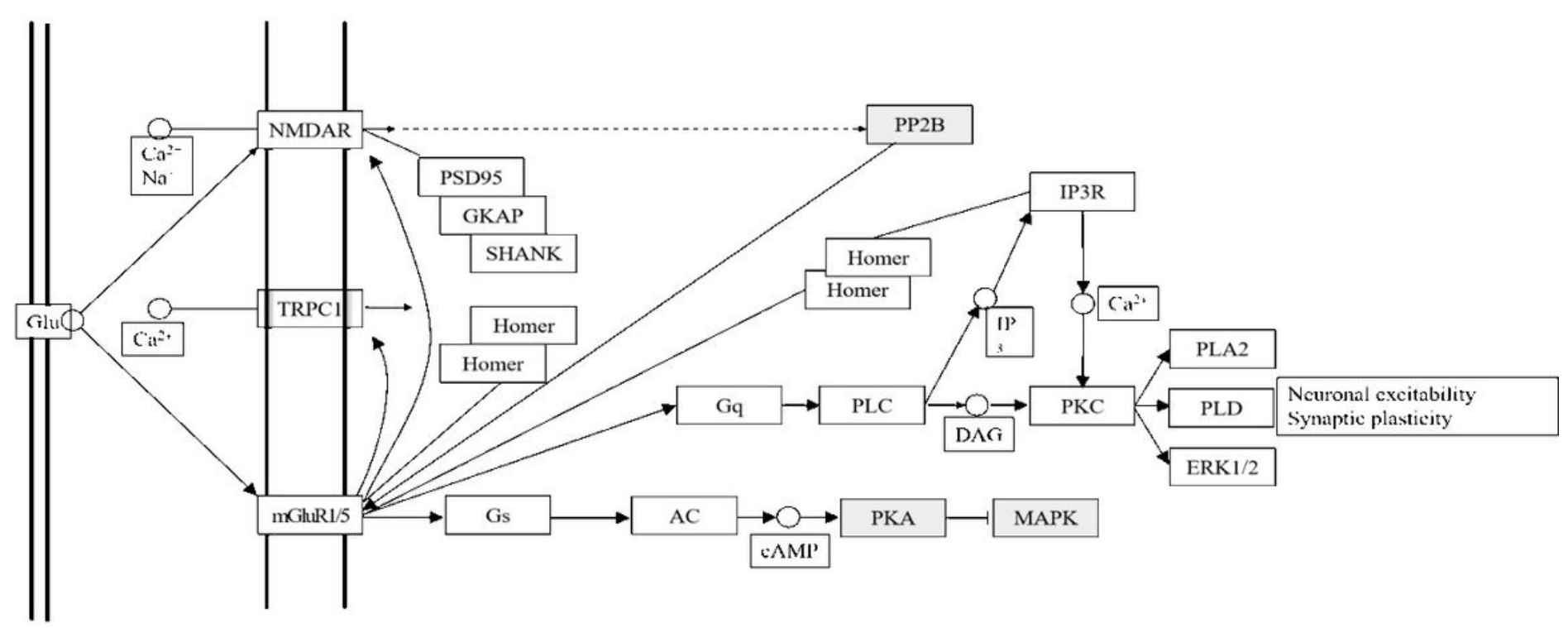

Figure 8

The evaluation of critical altered proteins that contribute to the pathological phenotypes of mice closely related to AD.; The protein expression level is given as the normalized protein abundance. Significant change levels of five proteins (PPP3CC, SNAPIN, RASA4, MERTK, and DNAJB1) were observed (A); proteins belonging to the glutamatergic synaptic regulating pathway and their potential interaction. Targets of CuraUltra-AD were colored in orange, and other protein targets in the pathway were colored in blue (B). 

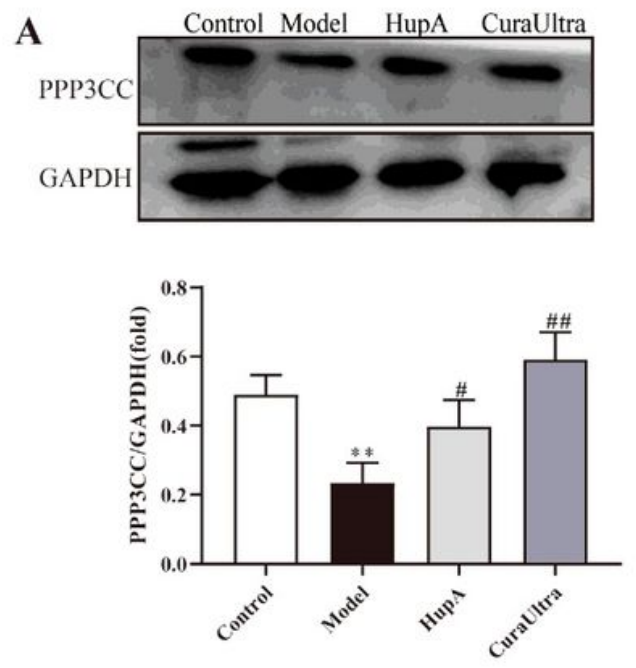

D
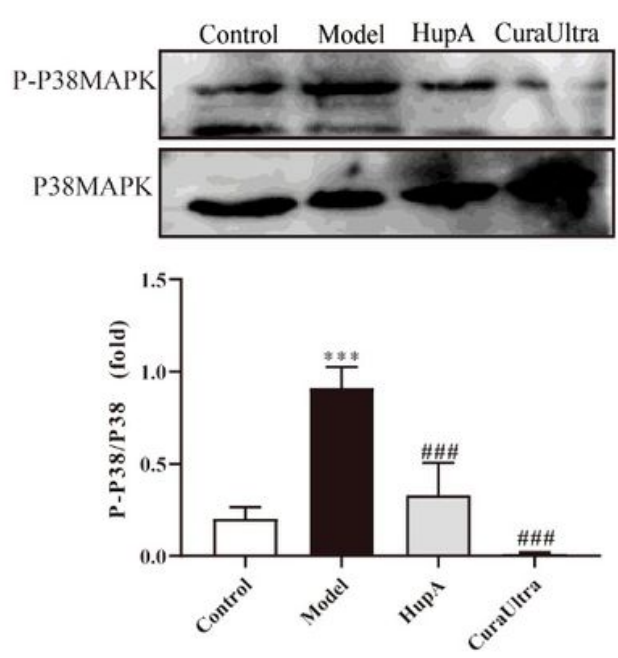
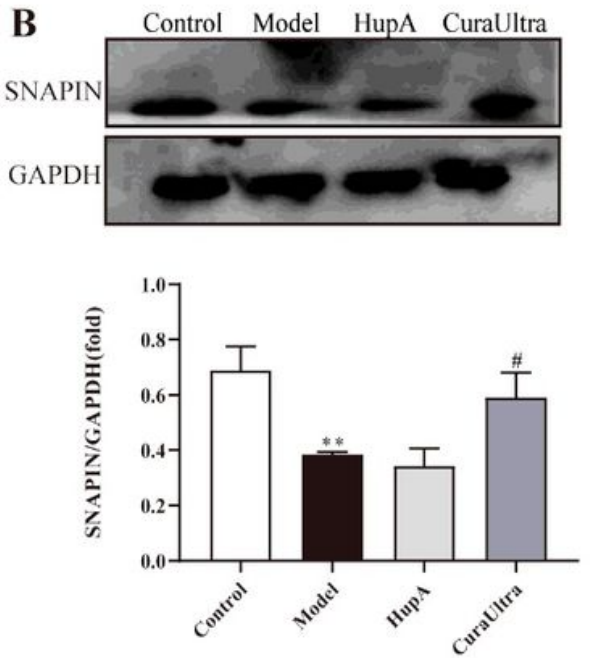

E

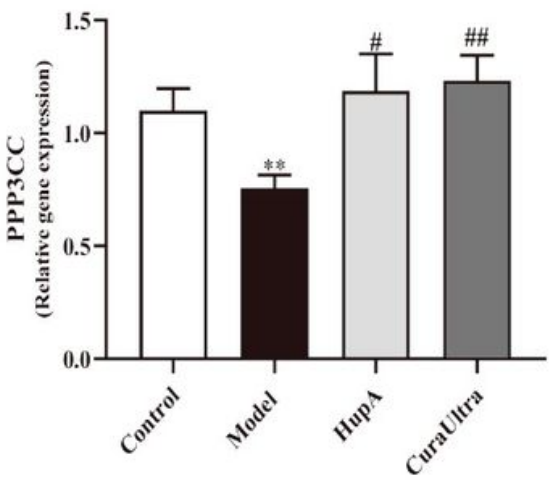

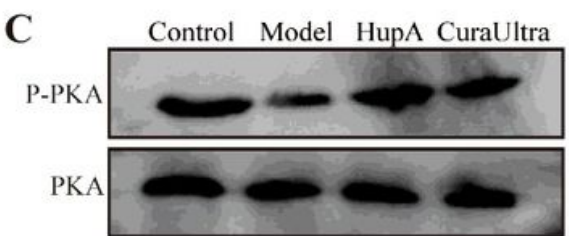

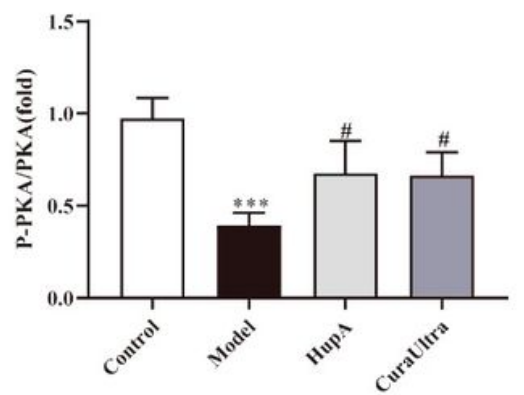

$\mathbf{F}$

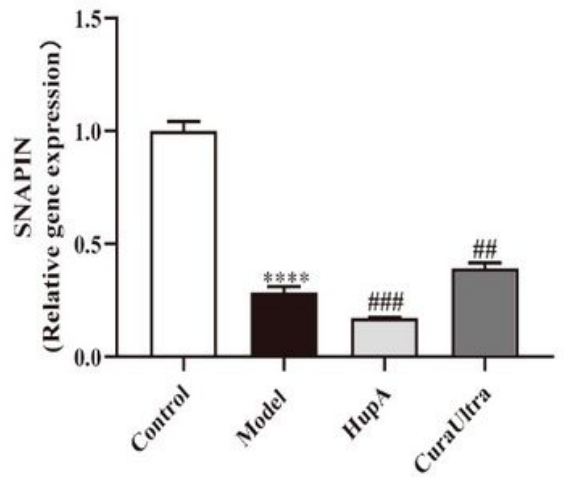

\section{Figure 9}

RT-qPCR and WB verified the change proteins. Representative images and the protein levels of PPP3CC, SNAPIN, PKA and P38MAPK in hippocampus tissues from mice were measured by Western blotting $(n=3)$ (A-D); Relative mRNA expression of PPP3CC and SNAPIN in hippocampus tissues were detected by real time-PCR analysis $(n=3)(E-F)$; Data were expressed as mean $\pm S E,{ }^{*} p<0.05,{ }^{*} p<0.01$ and ${ }^{* *} p<0.001$ compared to the control group; \#p $<0.05$, \#\#p $<0.01$ and \#\#\#p $<0.001$ compared to the scopolaminetreated group. 
A

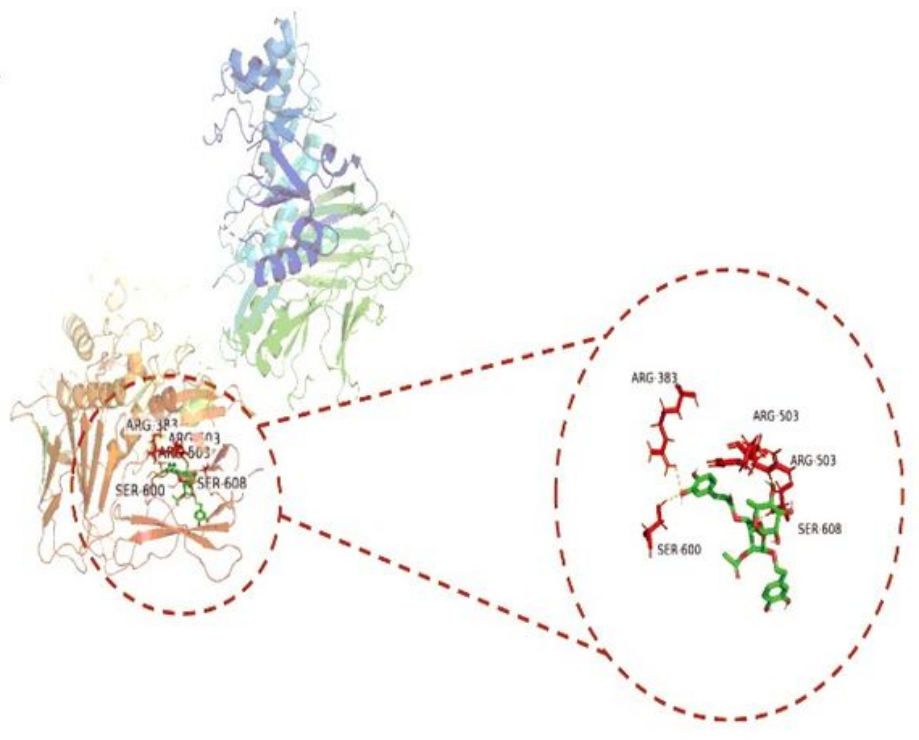

C

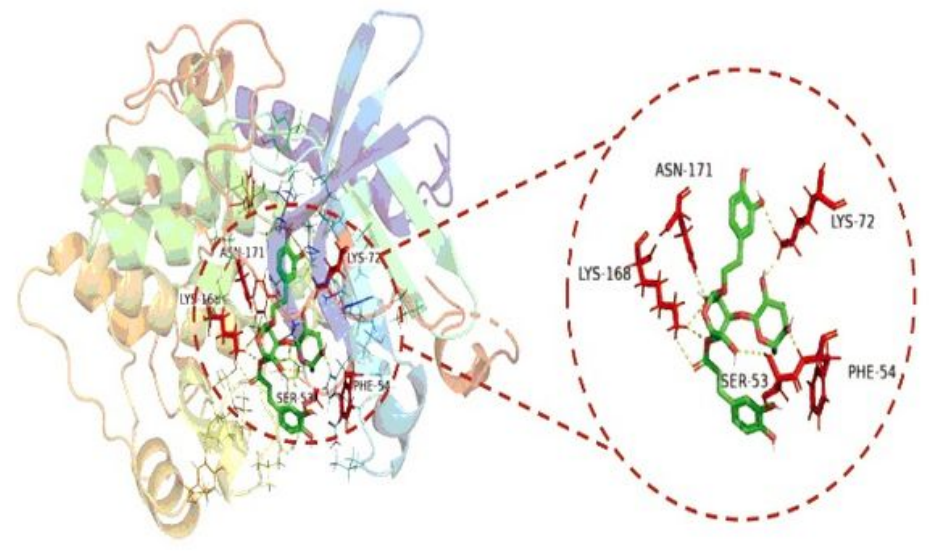

B

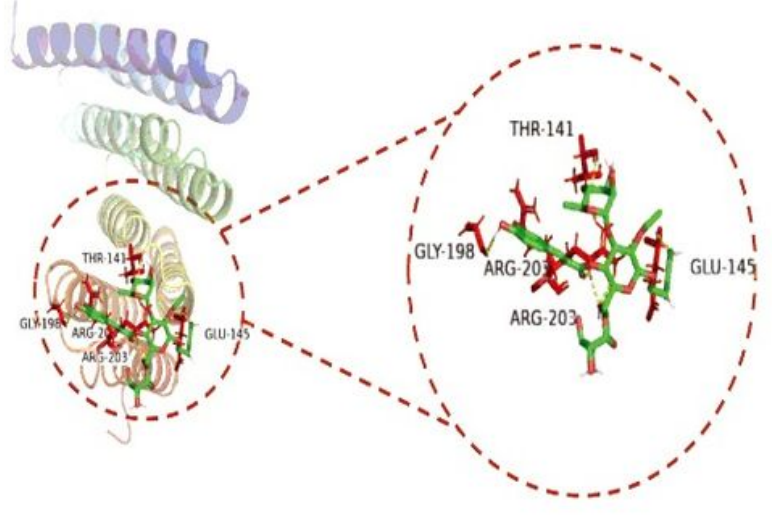

D

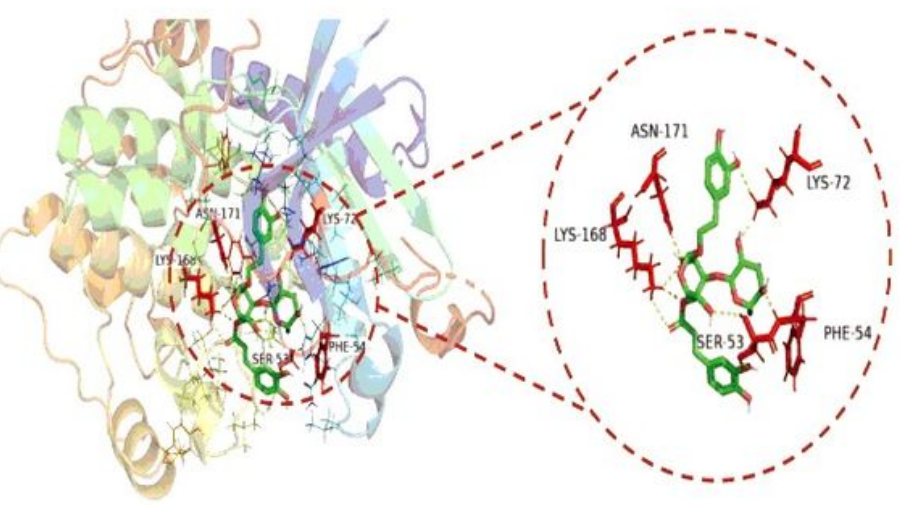

Figure 10

Analyses of target-compound docking simulation. 2-acetylacteoside acts on PPP3CC (A); Acteoside acts on PKA (B); Cistanoside A acts on P38MAPK (C); Parishin A acts on SNAPIN (D). A ball-and-stick represents the compound, a rainbow chain represents the target, a red stick represents the residues and hydrogen bonds are represented by a yellow dotted line. 


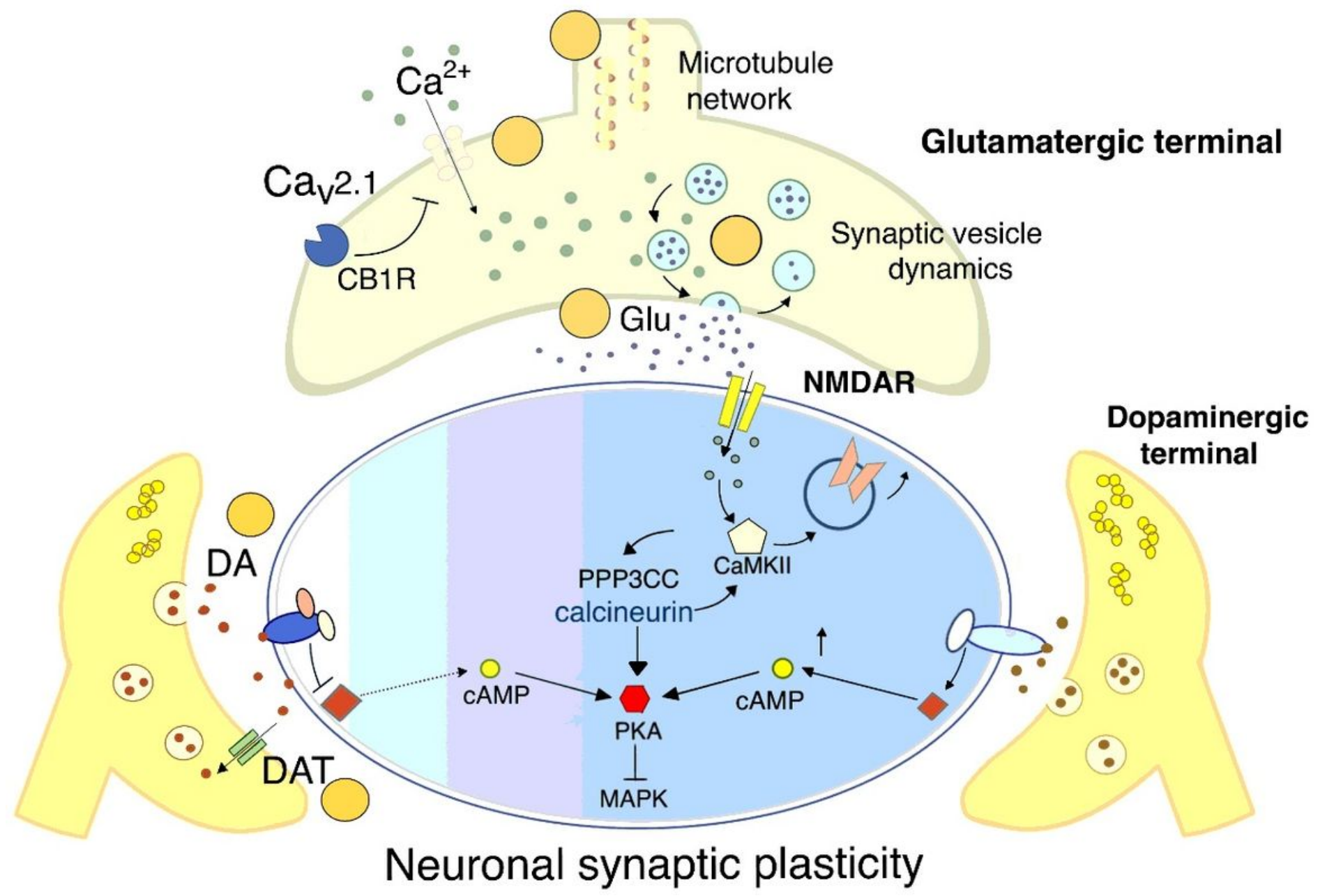

Figure 11

Schematic outline of the probable mechanisms behind CuraUltra-induced synaptic plasticity protection.

\section{Supplementary Files}

This is a list of supplementary files associated with this preprint. Click to download.

- SupplementaryTableS1.xlsx

- ThequantitativedataofWesternblot.xlsx

- FigureS1theimagesofWesternblot.pdf

- PCRdata.xIsx

- AcorusgramineusAitonextracts.pdf

- CistanchedeserticolaMaextrcts.pdf

- CurcumalongaL.extracts.pdf

- SupplementaryTableS2.xlsx 
- GastrodiaelataBlumeextracts.pdf

- SupplementaryTableS3.xlsx

- PolygalatenuifoliaWilldextracts.pdf

- RehmannialutinosaGaertn.DCextracts.pdf 